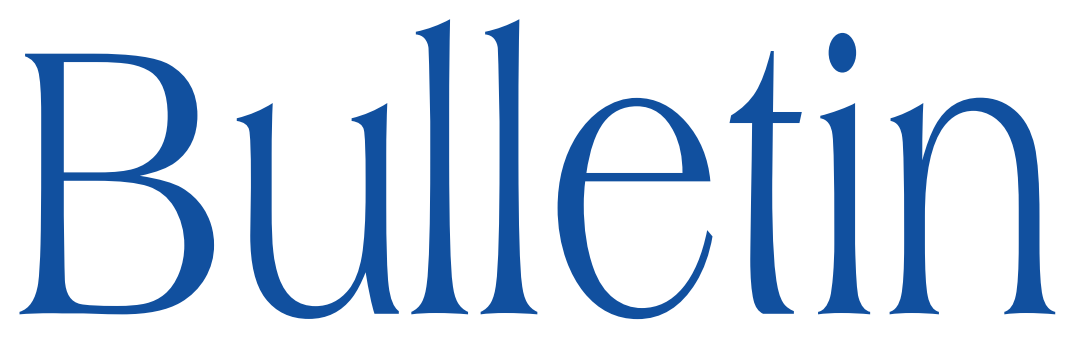

de la SOCIÉTÉ MATHÉMATIQUE DE FRANCE

\title{
SPECIALIZATION TO THE TANGENT CONE \\ AND WHITNEY EQUISINGULARITY
}

\author{
Arturo Giles Flores
}

Tome 141

Fascicule 2

2013 


\title{
SPECIALIZATION TO THE TANGENT CONE AND WHITNEY EQUISINGULARITY
}

\author{
By Arturo Giles Flores
}

\begin{abstract}
Let $(X, 0)$ be a reduced, equidimensional germ of an analytic singularity with reduced tangent cone $\left(C_{X, 0}, 0\right)$. We prove that the absence of exceptional cones is a necessary and sufficient condition for the smooth part $\mathfrak{X}^{0}$ of the specialization to the tangent cone $\varphi: \mathfrak{X} \rightarrow \mathbb{C}$ to satisfy Whitney's conditions along the parameter axis $Y$. This result is a first step in generalizing to higher dimensions Lê and Teissier's result for hypersurfaces of $\mathbb{C}^{3}$ which establishes the Whitney equisingularity of $X$ and its tangent cone under these conditions.
\end{abstract}

RÉSumé (Spécialisation sur le cône tangent et équisingularité à la Whitney)

Soit $(X, 0)$ un germe de singularité analytique complexe, réduit et équidimensionel tel que son cône tangent $\left(C_{X, 0}, 0\right)$ est réduit. On montre que l'absence des cônes exceptionnels est une condition nécessaire et suffisante pour que la partie lisse $\mathfrak{X}^{0}$ de la spécialisation sur le cône tangent $\varphi: \mathfrak{X} \rightarrow \mathbb{C}$ satisfasse les conditions de Whitney le long l'axe des paramètres $Y$. Ce résultat est un premier pas vers la généralisation aux dimensions supérieures du résultat de Lê et Teissier pour les hypersurfaces de $\mathbb{C}^{3}$ qui établit la équisingularité à la Whitney de $X$ et son cône tangent sous ces conditions.

Texte reçu le 17 février 2011, révisé le 3 septembre 2011 et accepté le 16 mars 2012.

Arturo Giles Flores, Institut de Mathématiques de Jussieu Url : http://www.math.jussieu.fr/ giles

- $\quad$-mail : giles@math.jussieu.fr

2010 Mathematics Subject Classification. - 14J17, 32S15.

Key words and phrases. - Equisingularity, Whitney conditions, Specialization to the tangent cone.

It is my pleasure to thank Bernard Teissier who patiently taught me everything I know about this subject. 


\section{Introduction}

The goal of this paper is to take a step in the study of the geometry of the specialization space $\varphi:(\mathfrak{X}, 0) \rightarrow(\mathbb{C}, 0)$ of a germ of reduced and $d$-dimensional singularity $(X, 0)$ to its tangent cone $C_{X, 0}$ from the point of view of Whitney equisingularity. The map $\varphi$ describes a flat family of analytic germs with a section $\mathfrak{X} \stackrel{\curvearrowleft}{\longrightarrow}: \sigma$, such that for each $t \in \mathbb{C}^{*}$ the germ $\left(\varphi^{-1}(t), \sigma(t)\right)$ is isomorphic to $(X, 0)$ and the special fiber is isomorphic to the tangent cone. This construction is essentially due to Gerstenhaber [4] in a more algebraic setting.

One would like to establish conditions on the strata of the canonical Whitney stratification of a reduced complex analytic germ which ensure the Whitney equisingularity of the germ and its tangent cone. In this paper we achieve the "codimension zero" part of this program.

The space $(\mathfrak{X}, 0) \rightarrow(\mathbb{C}, 0)$ has been used to study Whitney conditions in [10], and to study the structure of the set of limits of tangent spaces in [16] and [15]. In [16], the authors prove the existence of a finite family $\left\{V_{\alpha}\right\}$ of subcones of the reduced tangent cone $\left|C_{X, 0}\right|$ that determines the set of limits of tangent spaces to $X$ at 0 .

To be more specific, we fix an embedding $(X, 0) \subset\left(\mathbb{C}^{n+1}, 0\right)$ and build the normal/conormal diagram,

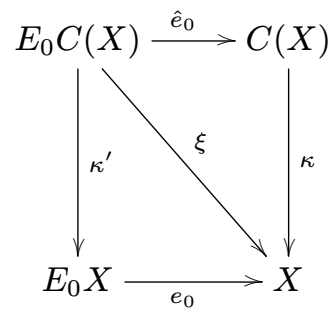

where $E_{0} X \subset X \times \mathbb{P}^{n}$ is the blowup of $X$ at the origin, $C(X) \subset X \times \check{\mathbb{P}}^{n}$ is the conormal space of $X$ whose fiber determines the set of limits of tangent spaces (see section 4), and $E_{0} C(X) \subset X \times \mathbb{P}^{n} \times \check{\mathbb{P}}^{n}$ is the blowup in $C(X)$ of the subspace $\kappa^{-1}(0)$; consider the irreducible decomposition of the reduced fiber $\left|\xi^{-1}(0)\right|=\bigcup D_{\alpha}$. The authors prove that the fiber $\xi^{-1}(0)$ is contained in the incidence variety $I \subset \mathbb{P}^{n} \times \check{\mathbb{P}}^{n}$ and that each $D_{\alpha}$ establishes a projective duality of its images $V_{\alpha} \subset \mathbb{P} C_{X, 0} \subset \mathbb{P}^{n}$ and $W_{\alpha} \subset \kappa^{-1}(0) \subset \check{\mathbb{P}}^{n}$.

In particular, the $V_{\alpha}$ 's that are not irreducible components of the tangent cone are called exceptional cones and they appear in $\mathfrak{X}$ as an obstruction to the $a_{f}$ stratification of the morphism $\mathfrak{X} \rightarrow \mathbb{C}$. They also prove that if the germ $(X, 0)$ is a cone itself, then it does not have exceptional cones. So a natural question arises, if a germ of an analytic singularity $(X, 0)$ does not have exceptional tangents, how close is it to being a cone?

TOME $141-2013-\mathrm{N}^{\mathrm{O}} 2$ 
A partial answer to this question was given in [15] in terms of Whitney equisingularity. The authors prove that for a surface $(S, 0) \subset\left(\mathbb{C}^{3}, 0\right)$ with reduced tangent cone $C_{S, 0}$, the absence of exceptional cones is a necessary and sufficient condition for it to be Whitney equisingular to its tangent cone.

The specialization space $(\mathfrak{X}, 0) \rightarrow(\mathbb{C}, 0)$ has a canonical section which picks the origin in each fiber (see section 2). Let $Y \subset \mathfrak{X}$ be given by this section and let $\mathfrak{X}^{0}$ be the non-singular part $\mathfrak{X}$. The main objective of this paper is to prove that if the germ $(X, 0)$ does not have exceptional cones and the tangent cone is reduced, then the couple $\left(\mathfrak{X}^{0}, Y\right)$ satisfies Whitney's conditions a) and b) at the origin.

\section{Specialization to the tangent cone.}

Let $(X, 0)$ be a reduced germ of an analytic singularity of pure dimension $d$, with tangent cone $C_{X, 0}$. Recall that the projectivized tangent cone can be defined as the exceptional divisor of the blowup of $X$ in 0 , and it is equivalent to considering the analytic "proj" of the graded algebra

$$
g r_{\mathfrak{m}} O_{X, 0}:=\bigoplus_{i \geq 0} \mathfrak{m}^{i} / \mathfrak{m}^{i+1}
$$

where $\mathfrak{m}$ is the maximal ideal of the analytic algebra $O_{X, 0}$ associated to the germ. Moreover, if we consider an embedding $(X, 0) \subset\left(\mathbb{C}^{n+1}, 0\right)$, the analytic algebra $O_{X, 0}$ is isomorphic to $\mathbb{C}\left\{z_{0}, \ldots, z_{n}\right\} / I$, where $I$ is an ideal, $g r_{\mathfrak{m}} O_{X, 0}$ is isomorphic to $\mathbb{C}\left[z_{0}, \ldots, z_{n}\right] / \operatorname{In}_{\mathfrak{M}} I$ where $\mathfrak{M}$ is the maximal ideal of $\mathbb{C}\left\{z_{0}, \ldots, z_{n}\right\}$, and the ideal $\operatorname{In}_{\mathfrak{M}} I$ is generated by all the initial forms with respect to the $\mathfrak{M}$-adic filtration of elements of $I$.

Let us suppose that the generators $\left\langle f_{1}, \ldots, f_{p}\right\rangle$ for $I$, were chosen in such a way that their initial forms generate the ideal $\operatorname{In}_{\mathfrak{M}} I$ defining the tangent cone. Note that the $f_{i}$ 's are convergent power series in $\mathbb{C}^{n+1}$, so if $m_{i}$ denotes the degree of the initial form of $f_{i}$, by defining

$$
F_{i}\left(z_{0}, \ldots, z_{n}, t\right):=t^{-m_{i}} f_{i}\left(t z_{0}, \ldots, t z_{n}\right)
$$

we obtain convergent power series, defining holomorphic functions on a suitable open subset $U$ of $\mathbb{C}^{n+1} \times \mathbb{C}$. Moreover, we can define the analytic algebra

$$
O_{\mathfrak{X}, 0}=\mathbb{C}\left\{z_{0}, \ldots, z_{n}, t\right\} /\left\langle F_{1}, \ldots, F_{p}\right\rangle
$$

with a canonical morphism $\mathbb{C}\{t\} \longrightarrow O_{\mathfrak{X}, 0}$ coming from the inclusion $\mathbb{C}\{t\} \hookrightarrow$ $\mathbb{C}\left\{z_{0}, \ldots, z_{n}, t\right\}$. Corresponding to this morphism of analytic algebras, we have the map germ $\varphi:(\mathfrak{X}, 0) \rightarrow(\mathbb{C}, 0)$ induced by the projection of $\mathbb{C}^{n+1} \times \mathbb{C}$ to the second factor. 
DeFinition 2.1. - The germ of analytic space over $\mathbb{C}$,

$$
\varphi:(\mathfrak{X}, 0) \rightarrow(\mathbb{C}, 0)
$$

is called the specialization of $(X, 0)$ to its tangent cone $\left(C_{X, 0}, 0\right)$.

There is another way of building this space that will allow us to derive some interesting properties. Let $E_{(0,0)} \mathbb{C}^{n+2}$ be the blowup of the origin of $\mathbb{C}^{n+2}$, where we now have the coordinate system $\left(z_{0}, \ldots, z_{n}, t\right)$. Let $W \subset E_{(0,0)} \mathbb{C}^{n+2}$ be the chart where the invertible ideal defining the exceptional divisor is generated by $t$, that is, in this chart the blowup map is given by $\left(z_{0}, \ldots, z_{n}, t\right) \mapsto$ $\left(t z_{0}, \ldots, t z_{n}, t\right)$.

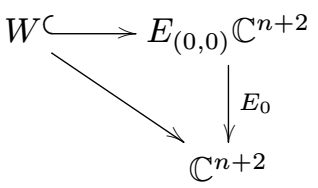

Lemma 2.2. - Let $X \times \mathbb{C} \subset \mathbb{C}^{n+2}$ be a small enough representative of the germ $(X \times \mathbb{C}, 0)$. If $(X \times \mathbb{C})^{\prime}$ denotes the strict transform of $(X \times \mathbb{C})$ in the blowup $E_{(0,0)} \mathbb{C}^{n+2}$, then the space $(X \times \mathbb{C})^{\prime} \cap W$ together with the map induced by the restriction of the map $E_{(0,0)} \mathbb{C}^{n+2} \rightarrow \mathbb{C}^{n+1} \times \mathbb{C} \rightarrow \mathbb{C}$ is isomorphic to the specialization space $\varphi: \mathfrak{X} \rightarrow \mathbb{C}$.

Proof. - We know that the strict transform $(X \times \mathbb{C})^{\prime}$ is isomorphic to the blowup of $X \times \mathbb{C}$ at the origin, and we are seeing it as a reduced analytic subvariety of $\mathbb{C}^{n+2} \times \mathbb{P}^{n+1}$. This means that the exceptional divisor $(X \times \mathbb{C})^{\prime} \cap$ $\left(\{0\} \times \mathbb{P}^{n+1}\right)$ is equal to $\mathbb{P}\left(C_{X, 0} \times \mathbb{C}\right)$, and so the ideal defining it is generated by the ideal defining the tangent cone $C_{X, 0}$ in $\mathbb{C}^{n+1}$, that is, the ideal of initial forms $\operatorname{In}_{\mathfrak{M}} I$. By hypothesis, $W \subset E_{(0,0)} \mathbb{C}^{n+2} \subset \mathbb{C}^{n+2} \times \mathbb{P}^{n+1}$ is set theoretically described by

$$
W=\left\{\left(t z_{0}, \ldots, t z_{n}, t\right),\left[z_{0}: \cdots: z_{n}: 1\right] \mid\left(z_{0}, \ldots, z_{n}, t\right) \in \mathbb{C}^{n+2}\right\}
$$

so in local coordinates the map $E_{0}$ restricted to $W$ is given by $\left(z_{0}, \ldots, z_{n}, t\right) \mapsto$ $\left(t z_{0}, \ldots, t z_{n}, t\right)$. Finally, since the ideal defining $X \times \mathbb{C}$ is generated in $\mathbb{C}\left\{z_{0}, \ldots, z_{n}, t\right\}$ by the ideal $I=\left\langle f_{1}, \ldots, f_{p}\right\rangle$ of $\mathbb{C}\left\{z_{0}, \ldots, z_{n}\right\}$ defining $X$ in $\mathbb{C}^{n+1}$, and since we have chosen the $f_{i}$ 's in such a way that their initial forms generate the ideal $\operatorname{In}_{\mathfrak{M}} I$, then the ideal defining the strict transform $(X \times \mathbb{C})^{\prime}$ in $W$ is given by

$$
\mathfrak{J} O_{W}=\left\langle t^{-m_{1}} f_{1}\left(t z_{0}, \ldots, t z_{n}\right), \ldots, t^{-m_{p}} f_{p}\left(t z_{0}, \ldots, t z_{n}\right)\right\rangle O_{W}
$$

that is, we find the same functions $F_{1}, \ldots, F_{p}$ which we used to define $\varphi: \mathfrak{X} \rightarrow$ $\mathbb{C}$. 
Proposition 2.3. - Let $\varphi: \mathfrak{X} \rightarrow \mathbb{C}$ be a small enough representative of the germ, then:

1. The morphism $\varphi$ is induced by the restriction of the projection $\mathbb{C}^{n+1} \times \mathbb{C} \rightarrow \mathbb{C}$ to the closed subspace defined by $\left(F_{1}, \ldots, F_{p}\right)$, and it is faithfully flat.

2. The special fiber $\mathfrak{X}(0):=\varphi^{-1}(0)$ is isomorphic to the tangent cone $C_{X, 0}$.

3. The analytic space $\mathfrak{X} \backslash \varphi^{-1}(0)$ is isomorphic to $X \times \mathbb{C}^{*}$ as an analytic space over $\mathbb{C}^{*}$. In particular, for every $t \in \mathbb{C}^{*}$, the germ $\left(\varphi^{-1}(t),\{0\} \times t\right)$ is isomorphic to $(X, 0)$.

4. The germ $(\mathfrak{X}, 0)$ is reduced and of pure dimension $d+1$.

that is, we have produced a 1-parameter flat family of germs of analytic spaces specializing $(X, 0)$ to $\left(C_{X, 0}, 0\right)$.

Proof. - First of all, note that the inclusion $\mathbb{C}\{t\} \hookrightarrow \mathbb{C}\left\{z_{0}, \ldots, z_{n}, t\right\}$ can be seen as the stalk map at the origin of the holomorphic map defined by the linear projection onto the last coordinate $\mathbb{C}^{n+1} \times \mathbb{C} \rightarrow \mathbb{C}$. This implies that $\varphi$ is just the restriction to $\mathfrak{X}$ of this projection.

Now, to prove the (faithful) flatness of $\varphi$ we must prove that $O_{\mathfrak{X}, 0}$ is faithfully flat as a $\mathbb{C}\{t\}$ module, but by [5, Prop. B.3.3, p. 404] flat implies faithfully flat for local rings, and by [6, Corollary 7.3.5, p. 390$] O_{\mathfrak{X}, 0}$ is flat if and only if it is torsion free. In other words all we have to prove is that $t$ is not a zero divisor in $O_{\mathfrak{X}, 0}$.

But by Lemma 2.2, $\mathfrak{X}$ is isomorphic to an open subset of the blowup of $X \times \mathbb{C}$ along the subspace $\{0\} \times \mathbb{C}$, where the ideal of the exceptional divisor is invertible, generated by $t$. Thus, by definition of blowup, $t$ is not a zero divisor, $\mathfrak{X}$ is of pure dimension $d+1$ (the dimension of $X \times \mathbb{C}$ ), and since the blowup of a reduced space remains reduced then $\mathfrak{X}$ is reduced.

The biholomorphism of the map induced by the isomorphism

$$
\phi: \mathbb{C}^{n+1} \times \mathbb{C}^{*} \rightarrow \mathbb{C}^{n+1} \times \mathbb{C}^{*} \text { defined by }(\underline{z}, t) \mapsto(t \underline{z}, t)
$$

is also a direct consequence of Lemma 2.2. It maps $\mathfrak{X} \backslash \mathfrak{X}(0)$ onto $\mathfrak{X} \times \mathbb{C}^{*}$, and for each $t \neq 0$ the fiber $\mathfrak{X}(t)$ is mapped bilohomorphically onto $X \times\{t\}$.

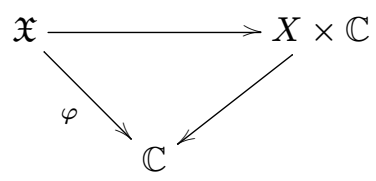

Finally, the fact that the special fiber $\mathfrak{X}(0)$ is isomorphic to the tangent cone can be read directly from the analytic functions $F_{1}, \ldots, F_{p}$ defining $\mathfrak{X}$, since when setting $t=0$ we have the initial forms $F_{i}(z, 0)=f_{m_{i}}$ which by hypothesis generate the ideal defining the tangent cone in $\mathbb{C}^{n+1}$. 
A more detailed description of this space, relating it to a generalized Rees algebra and interpreting the space thus obtained as the open set of the blowup (2.2) of $X \times \mathbb{C}$ at the origin can be found in [15, p. 428-430] for surfaces, and [16, p. 556-557], or [10, p. 200-202] in the general case.

\section{REMARK 2.4. - Note that:}

1. The map $\phi: \mathfrak{X} \rightarrow X \times \mathbb{C}$ from Proposition 2.3 is defined everywhere and maps the entire fiber $\mathfrak{X}(0)$ to the origin in $X \times \mathbb{C}$.

2. If we denote by $\mathfrak{X}(t)^{0}$ the non-singular part of the fiber, the open dense subset $\bigcup_{t} \mathfrak{X}(t)^{0} \subset \mathfrak{X}$ is called the relative smooth locus of $\mathfrak{X}$ with respect to $\varphi$ and it is equal to $\mathfrak{X}^{0} \backslash \operatorname{Sing} \mathfrak{X}(0)$.

Lemma 2.5. - Let $X=\cup_{j=1}^{r} X_{j}$ be the irreducible decomposition of $X$. Then, the specialization $\mathfrak{X}_{j}$ of $X_{j}$ is an analytic subspace of $\mathfrak{X}$, and the following diagram commutes.

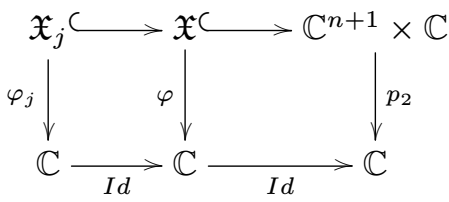

In particular, $\mathfrak{X}=\cup_{j=1}^{r} \mathfrak{X}_{j}$ is the irreducible decomposition of $\mathfrak{X}$.

Proof. - Note that $X_{j}$ is a proper analytic subspace of $X$ for all $j \geq 1$, so we have a strict inclusion of their corresponding ideals in $O_{n+1}:=\mathbb{C}\left\{z_{0}, \ldots, z_{n}\right\}$, namely $I \subset J$, from which we immediately obtain that $\operatorname{In}_{\mathfrak{M}} I \subset I n_{\mathfrak{M}} J$ or equivalently $C_{X_{j}, 0} \subset C_{X, 0}$.

Now let us take as before, generators for $I$, say $I=\left\langle f_{1}, \ldots, f_{p}\right\rangle$, in such a way that their initial forms generate the ideal defining the tangent cone $I n_{\mathfrak{M}} I$, and doing the same for $J$, we get $J=\left\langle g_{1}, \ldots, g_{s}\right\rangle$ and $I n_{\mathfrak{M}} J=\left\langle i n_{\mathfrak{M}} g_{1}, \ldots, i n_{\mathfrak{M}} g_{s}\right\rangle$. But the previous inclusions tell us that we can choose as generators for $J=$ $\left\langle f_{1}, \ldots, f_{p}, g_{1}, \ldots, g_{s}\right\rangle$, and still get that their initial forms generate the ideal $I n_{\mathfrak{M}} J=\left\langle i n_{\mathfrak{M}} f_{i}, i n_{\mathfrak{M}} g_{j}\right\rangle$.

So finally, to build the specialization spaces $\mathfrak{X}$ and $\mathfrak{X}_{j}$ as we did before, we define the convergent series in $O_{n+2}, F_{i}(z, t)=t^{-m_{i}} f_{i}\left(t z_{0}, \ldots, t z_{n}\right)$ and $G_{j}(z, t)=$ $t^{-m_{j}} g_{j}\left(t z_{0}, \ldots, t z_{n}\right)$, that give us the embedding $\mathfrak{X}:=V\left(F_{1}, \ldots, F_{p}\right) \subset \mathbb{C}^{n+1} \times$ $\mathbb{C}$ and the embedding $\mathfrak{X}_{j}:=V\left(F_{1}, \ldots, F_{p}, G_{1}, \ldots, G_{s}\right) \subset \mathbb{C}^{n+1} \times \mathbb{C}$. Moreover, since $\left\langle F_{1}, \ldots, F_{p}\right\rangle \subset\left\langle F_{i}, G_{j}\right\rangle$ then we have a closed embedding $\mathfrak{X}_{j} \subset \mathfrak{X}$ compatible with the projection to the $t$ axis. 
And even more, since with respect to this embedding of $\mathfrak{X}$ in $\mathbb{C}^{n+1} \times \mathbb{C}$, the isomorphism $\phi$ is of the form:

$$
\begin{aligned}
\phi: \mathfrak{X} \backslash \varphi^{-1}(0) & \longrightarrow X \times \mathbb{C}^{*} \\
\left(z_{0}, \ldots, z_{n}, t\right) & \longmapsto\left(t z_{0}, \ldots, t z_{n}, t\right)
\end{aligned}
$$

We also have compatibility with the isomorphism, that is $\phi_{j}=\left.\phi\right|_{\mathfrak{X}_{j}}$.

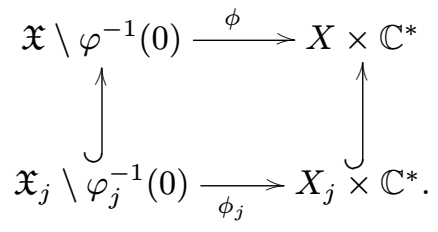

REMARK 2.6. - 1. For an analytic subspace $Y \subset X$ we can mimic the construction of Lemma 2.2 to build the specialization space $\varphi: \mathfrak{X} \rightarrow \mathbb{C}$ where we still have that the fiber $(\mathfrak{X}(t),(0, t))_{t \neq 0}$ is isomorphic to the germ $(X, 0)$, but this time the special fiber $(\mathfrak{X}(0),(0,0))$ is isomorphic to the normal cone $\left(C_{X, Y}, 0\right)$. The map $\varphi$ is again faithfully flat.

2. If $Y \subset X$ is a linear subspace defined by the ideal $J=\left\langle z_{0}, \ldots z_{n-s}\right\rangle \mathbb{C}\left\{z_{0}, \ldots, z_{n-s}, y_{1}, \ldots, y_{s}\right\}$ then we can choose analytic functions $f_{1}, \ldots, f_{p}$ such that they generate the ideal $I$ defining $X$ in $\mathbb{C}^{n+1}$, and their initial forms $f_{m_{i}}=i n_{J} f_{i}$ generate the ideal of initial forms $i n_{J} I$. In this case the ideal generated by the analytic functions $F_{i}(z, y, t)=t^{-m_{i}} f_{i}\left(t z_{0}, \ldots, t z_{n-t}, y_{1}, \ldots, y_{s}\right)$ will be the ideal defining the space $\mathfrak{X}$ in $\mathbb{C}^{n+1} \times \mathbb{C}$, where $m_{i}$ is equal to $\nu_{Y} f_{i}$.

\section{The Relative Nash Modification of $\mathfrak{X}$}

Let us take a representative $\varphi: \mathfrak{X} \rightarrow \mathbb{C}$ of the germ $\varphi:(\mathfrak{X}, 0) \rightarrow(\mathbb{C}, 0)$, and consider the map:

$$
\begin{aligned}
\gamma_{\varphi}: \mathfrak{X}_{\varphi}^{\circ} & \longrightarrow G r(d, n+1) \\
(z, t) & \longrightarrow T_{(z, t)} X_{\varphi}^{\circ}(t)
\end{aligned}
$$

where $\mathfrak{X}_{\varphi}^{\circ}$ denotes the relative smooth locus of $\mathfrak{X}$ with respect to $\varphi, G r(d, n+1)$ corresponds to the grassmannian of directions of $d$-planes of the hyperplane $\{t=0\} \subset \mathbb{C}^{n+1} \times \mathbb{C}$, and $T_{(z, t)} \mathfrak{X}_{\varphi}^{\circ}(t)$ denotes the tangent space to the fiber $\mathfrak{X}_{\varphi}^{\circ}(t)$ at the point $(z, t)$. The closure $\mathcal{N}_{\varphi} \mathfrak{X}$ of the graph of $\gamma_{\varphi}$ in $\mathfrak{X} \times G r(d, n+1)$ is an analytic space of dimension $d+1$, which is known as the relative Nash modification of $\varphi: \mathfrak{X} \rightarrow \mathbb{C}$.

A. Nobile proved in [11, Thm 1, p. 299] that the Nash modification is a blowup. The main ingredient of his proof is the Plücker embedding of the 
grassmannian $G(d, n+1)$ in the projective space $\mathbb{P}^{N}$, where $N=\left(\begin{array}{c}n+1 \\ d\end{array}\right)$. Minor modifications of the proof immediately gives us an analogous result for the relative case. We will only state it in the case of $\varphi: \mathfrak{X} \rightarrow \mathbb{C}$.

Lemma 3.1. - The relative Nash modification $\nu_{\varphi}: \mathcal{N}_{\varphi} \mathfrak{X} \rightarrow \mathfrak{X}$ is locally a blowup with center a suitable ideal $J_{\varphi} \subset O_{\mathfrak{X}}$. Moreover, if $(\mathfrak{X}, 0)$ is a complete intersection of dimension $n+2-p$ then we may take the ideal $J_{\varphi} \subset O_{\mathfrak{X}}$ to be the relative Jacobian ideal, generated by the $p \times p$ minors of the relative Jacobian matrix $\left[D_{\varphi} F\right]=\left[\frac{\partial F_{i}}{\partial z_{j}}\right]_{j=0 \ldots n}^{i=1 \ldots p}$. (We are omitting the partial derivatives with respect to the parameters, which in this case correspond to the t-coordinate).

Proof. - Given integers $n+1 \geq r>0, p \geq n+1-r$ and a $p \times(n+1)$ matrix $A$, let $S$ (resp. $\left.S^{\prime}\right)$ denote the set of increasing sequences of $n+1-r$ positive integers less than $p+1$ (resp. $n+2)$; if $\alpha=\left(\alpha_{1}, \ldots, \alpha_{n+1-r}\right) \in S$, $\beta=\left(\beta_{1}, \ldots, \beta_{n+1-r}\right) \in S^{\prime}$, then $M_{\alpha \beta}$ will denote the minor of $A$ obtained by considering the rows determined by $\alpha$ and the columns determined by $\beta$.

Following the proof of Nobile, let $\mathfrak{X}=\bigcup_{j=1}^{k} \mathfrak{X}_{j}$ be the irreducible decomposition of a small enough representative of $(\mathfrak{X}, 0)$. Let $\left[D_{\varphi} F\right]=\left[\frac{\partial F_{i}}{\partial z_{j}}\right]_{j=0 \ldots n}^{i=1 \ldots p}$ be the relative Jacobian matrix of the map $\varphi: \mathfrak{X} \rightarrow \mathbb{C}$. By construction, there is an open dense set $U \subset \mathfrak{X}$, such that for every point $\left(z_{0}, t_{0}\right)$ in $U$ the matrix $\left[D_{\varphi} F\left(z_{0}, t_{0}\right)\right]$ has rank $n+1-d$. Since $\mathfrak{X}$ is reduced, each irreducible component $\mathfrak{X}_{i}$ is reduced and so for each $i=1, \ldots, k$ there exists a pair $\left(\alpha^{i}, \beta^{i}\right) \in S \times S^{\prime}$ such that the $(n+1-d) \times(n+1-d)$ minor $M_{\alpha^{i} \beta^{i}}$ of $\left[D_{\varphi} F\right]$ does not vanish identically on $\mathfrak{X}_{i}$. For each $i=1, \ldots, k$, fix $H_{i} \in O_{\mathfrak{X}, 0}$ such that $H_{i}=0$ on $\bigcup_{j \neq i} \mathfrak{X}_{j}$, and $H_{i} \neq 0$ on $\mathfrak{X}_{i}$. For each $\beta \in S^{\prime}$ define the function $G_{\beta}=\sum_{i=1}^{k} H_{i} M_{\alpha^{i} \beta} \in O_{\mathfrak{X}, 0}$, and consider the ideal $J_{\varphi} \subset O_{\mathfrak{X}, 0}$ generated by the $G_{\beta}$ 's.

Note that the analytic subset $V\left(J_{\varphi}\right)$ of $\mathfrak{X}$ defined by the ideal $J_{\varphi}$ contains the relative singular locus of $\varphi: \mathfrak{X} \rightarrow \mathbb{C}$. Moreover, the open set $W:=\mathfrak{X} \backslash V\left(J_{\varphi}\right)$ is dense in $\mathfrak{X}$. Finally if we build a representative of this blowup using the functions $G_{\beta}$, we will have it as an analytic subspace of $\mathfrak{X} \times \mathbb{P}^{N}$, with $N=$ $\left(\begin{array}{c}n+1 \\ n+1-d\end{array}\right)-1=\left(\begin{array}{c}n+1 \\ d\end{array}\right)-1$, and for a point $(z, t) \in \mathfrak{X}_{i} \cap W$ we have that: $\left[G_{\beta}(z, t)\right]=\left[\sum_{j=1}^{k} H_{j}(z, t) M_{\alpha^{j} \beta}(z, t)\right]=\left[H_{i}(z, t) M_{\alpha^{i} \beta}(z, t)\right]=\left[M_{\alpha^{i} \beta}(z, t)\right] \in \mathbb{P}^{N}$ 
which corresponds to the coordinates of the tangent space $T_{(z, t)} X_{\varphi}^{\circ}(t)$ for the Plücker embedding of the grassmannian $G(d, n+1)$, in the projective space $\mathbb{P}^{N}$.

This lemma allows us to establish the following relation between the Nash modification of $X$ and the relative Nash modification of $\mathfrak{X}$.

Proposition 3.2. - There exists a natural surjective morphism $\Gamma: \mathcal{N}_{\varphi} \mathfrak{X} \rightarrow$ $\mathcal{N} X$, making the following diagram commute:

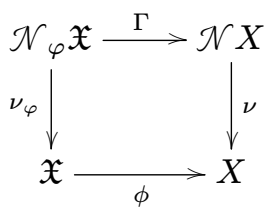

Proof. - Algebraically, this results from the universal property of the blowup $\nu: \mathcal{N} X \rightarrow X$. We start with the diagram:

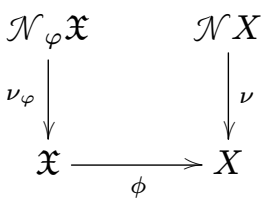

where the map $\phi$ is defined by $\left(z_{0}, \ldots, z_{n}, t\right) \rightarrow\left(t z_{0}, \ldots, t z_{n}\right)$, and so it induces a morphism of analytic algebras $\phi^{*}: O_{X, 0} \rightarrow O_{\mathfrak{X}, 0}$ defined by $z_{i} \rightarrow t z_{i}$.

Recall that the ideal of the germ $(\mathfrak{X}, 0)$ is generated by the series $F_{i}(z, t)=$ $t^{-m_{i}} f_{i}(t z) \in \mathbb{C}\left\{z_{0}, \ldots, z_{n}, t\right\}, i=1, \ldots, p$, where the series $f_{j} \in \mathbb{C}\left\{z_{0}, \ldots, z_{n}\right\}$ are such that they generate the ideal of $(X, 0)$ in $\left(\mathbb{C}^{n+1}, 0\right)$ and their initial forms generate the ideal of $\left(C_{X, 0}, 0\right)$.

By [11, Thm 1, p. 299] there exists an ideal $J O_{X, 0}$ whose blowup is isomorphic to the Nash modification of $X$. We have to prove that the ideal $\phi^{*}(J) O_{\mathfrak{X}, 0}$ is locally invertible when pulled back to $\mathcal{N}_{\varphi} \mathfrak{X}$.

Let $X=\bigcup_{j=1}^{k} X_{j}$ be the irreducible decomposition of a small enough representative of $(X, 0)$. Then the irreducible decomposition of a small enough representative of the germ $(\mathfrak{X}, 0)$ is of the form $\bigcup_{j=1}^{k} \mathfrak{X}_{j}$, where for each $j$ the space $\mathfrak{X}_{j}$ is isomorphic to the specialization space of the $X_{j}$ component to its tangent cone $C_{X_{j}, 0}$. Now, by [11, Thm 1, p. 299] the ideal $J \subset O_{X, 0}$ can be constructed in the following way (see the proof of 3.1 for more details and notation): For each $i=1, \ldots, k$ there exists a pair $\left(\alpha^{i}, \beta^{i}\right) \in S \times S^{\prime}$ such that the $(n+1-d) \times(n+1-d)$ minor $\mu_{\alpha^{i} \beta^{i}}$ of the Jacobian matrix $[D f]$ does not vanish identically on $X_{i}$. Then for each $i=1, \ldots, k$, choose a function $h_{i} \in O_{X, 0}$ such that $h_{i}=0$ on $\bigcup_{j \neq i} X_{j}$, and $h_{i} \neq 0$ on $X_{i}$. By taking powers of the $h_{i}$ 's if necessary we can assume they are all of the same order $\gamma$. Finally for each 
$\beta \in S^{\prime}$ define the function $g_{\beta}=\sum_{i=1}^{k} h_{i} \mu_{\alpha^{i} \beta} \in O_{X, 0}$, and define $J$ as the ideal generated by the $g_{\beta}$ 's.

Consider an $(n+1-d) \times(n+1-d)$ minor $\mu_{\alpha \beta}$ of the Jacobian matrix $[D f]$

$$
\mu_{\alpha, \beta}=\left|\begin{array}{ccc}
\frac{\partial f_{\alpha_{1}}}{\partial z_{\beta_{1}}}(z) & \cdots & \frac{\partial f_{\alpha_{1}}}{\partial z_{\beta_{n+1}-d}}(z) \\
\vdots & \vdots & \vdots \\
\frac{\partial f_{\alpha_{n+1-d}}}{\partial z_{\beta_{1}}}(z) & \cdots & \frac{\partial f_{\alpha_{n+1-d}}}{\partial z_{\beta_{n+1}-d}}(z)
\end{array}\right|
$$

Then, from the equalities $\phi^{*}\left(\frac{\partial f_{i}}{\partial z_{j}}(z)\right)=\frac{\partial f_{i}}{\partial z_{j}}(t z)$, and $\frac{\partial f_{i}}{\partial z_{j}}(t z)=t^{m_{i}-1} \frac{\partial F_{i}}{\partial z_{j}}(z, t)$, we have that the minor $\mu_{\alpha \beta}$ is mapped under $\phi^{*}$ to:

$$
\begin{aligned}
\phi^{*}\left(\mu_{\alpha \beta}\right) & =\left|\begin{array}{ccc}
t^{m_{\alpha_{1}}-1} \frac{\partial F_{\alpha_{1}}}{\partial z_{\beta_{1}}}(z, t) & \cdots & t^{m_{\alpha_{1}}-1} \frac{\partial F_{\alpha_{1}}}{\partial z_{\beta_{n+1}-d}}(z, t) \\
\vdots & \vdots & \vdots \\
t^{m_{\alpha_{n+1-d}}-1} \frac{\partial F_{\alpha_{n+1}-d}}{\partial z_{\beta_{1}}}(z, t) & \cdots & t^{m_{\alpha_{n+1}-d}}-\frac{\partial F_{\alpha_{n+1}-d}}{\partial z_{\beta n+1-d}}(z, t)
\end{array}\right| \\
= & t^{\left(\sum_{1}^{n+1-d} m_{\alpha_{i}}\right)-(n+1-d)}\left|\begin{array}{ccc}
\frac{\partial F_{\alpha_{1}}}{\partial z_{\beta_{1}}}(z, t) & \cdots & \frac{\partial F_{\alpha_{1}}}{\partial z_{\beta_{n+1}-d}}(z, t) \\
\vdots & \vdots & \vdots \\
\frac{\partial F_{\alpha_{n+1}-d}}{\partial z_{\beta_{1}}}(z, t) & \cdots & \frac{\partial F_{\alpha_{n+1}-d}}{\partial z_{\beta n+1-d}}(z, t)
\end{array}\right| \\
= & t^{\left(\sum_{1}^{n+1-d} m_{\alpha_{i}}\right)-(n+1-d)} M_{\alpha \beta}
\end{aligned}
$$

where $M_{\alpha \beta}$ is the $(n+1-d) \times(n+1-d)$ minor of the relative Jacobian matrix $\left[D_{\varphi} F\right]$.

If we define $H_{i} \in O_{\mathfrak{X}, 0}$ by $H_{i}(z, t)=t^{-\gamma} h_{i}(t z)$, then each $H_{i}$ satisfies that $H_{i}=0$ on $\bigcup_{j \neq i} \mathfrak{X}_{j}$, and $H_{i} \neq 0$ on $\mathfrak{X}_{i}$ and so for each $\beta \in S^{\prime}$ we have that

$\phi^{*}\left(g_{\beta}\right)=\sum_{i=1}^{k} \phi^{*}\left(h_{i}\right) \phi^{*}\left(\mu_{\alpha^{i} \beta}\right)=t^{\left(\gamma+\left(\sum_{1}^{n+1-d} m_{\alpha_{i}}\right)-(n+1-d)\right)} \sum_{i=1}^{k} H_{i} M_{\alpha^{i} \beta}=t^{r} G_{\beta}$

and so

$$
\phi^{*}(J) O_{\mathfrak{X}, 0}=\left\langle t^{r}\right\rangle J_{\varphi} O_{\mathfrak{X}, 0}
$$

where by the proof of $3.1 J_{\varphi} O_{\mathfrak{X}, 0}$ is an ideal whose blowup is isomorphic to the relative Nash modification $\mathcal{N}_{\varphi} \mathfrak{X}$. But by definition of the blowup, the ideal $J_{\varphi} O_{\mathfrak{X}, 0}$ is locally invertible when pulled back to $\mathcal{N}_{\varphi} \mathfrak{X}$. It follows that after multiplication by the invertible ideal $\left\langle t^{r}\right\rangle$ in $O_{\mathfrak{X}, 0}$, it will remain locally invertible when pulled back to $\mathcal{N}_{\varphi} \mathfrak{X}$.

Finally, note that for the diagram to be commutative, the morphism $\Gamma$ must map the point $\left(z, t, T_{(z, t)} \mathfrak{X}_{\varphi}^{\circ}(t)\right) \in \mathcal{N}_{\varphi} \mathfrak{X}$ to the point $\left(t z, T_{(t z)} X^{\circ}\right) \in \mathcal{N} X$. That is the tangent space $T_{(z, t)} \mathfrak{X}_{\varphi}^{\circ}(t)$ to the fiber $\mathfrak{X}(t)$ is canonically identified with the tangent space $T_{(t z)} X^{\circ}$ to $X$ at the corresponding points. As it should be

TOME $141-2013-\mathrm{N}^{\mathrm{O}} 2$ 
since we know that the restriction of the map $\phi$ to any fiber $(\mathfrak{X}(t),(0, t))$ for $t \neq 0$ is an isomorphism with $(X, 0)$.

\section{The conormal space and relative conormal space of $\mathfrak{X}$.}

Let $\mathfrak{X} \subset \mathbb{C}^{n+2}$ be a representative of the germ $(\mathfrak{X}, 0)$. Recall that the projectivized conormal space of $\mathfrak{X}$ in $\mathbb{C}^{n+2}$ is an analytic space $C(\mathfrak{X}) \subset \mathfrak{X} \times \check{\mathbb{P}}^{n+1}$, together with a proper analytic map $\kappa_{\mathfrak{X}}: C(\mathfrak{X}) \rightarrow \mathfrak{X}$, where the fiber over a smooth point $p \in \mathfrak{X}$ is the set of tangent hyperplanes, that is the hyperplanes $H$ containing the direction of the tangent space $T_{p} \mathfrak{X}$. The space $C(\mathfrak{X})$, depends on the embedding, however the fiber $\kappa_{\mathfrak{X}}^{-1}(p)$ allows us to recover the fiber of the Nash modification, which is independent of the embedding. Up to now we have:

$$
\mathcal{N} \mathfrak{X} \subset \mathfrak{X} \times G(d+1, n+2) \subset \mathfrak{X} \times \mathbb{P}^{N}
$$

But we know that the grassmannian $G(d+1, n+2)$ is isomorphic to the grassmannian $G(n+1-d, n+2)$ and the isomorphism is given by sending a $d+1$-plane $T$ to the $n+1-d$-plane $L$ of linear functionals in $\breve{C}^{n+2}$ that vanish on $T$. With this isomorphism, we have:

$$
\mathcal{N} \mathfrak{X} \subset \mathfrak{X} \times G(n+1-d, n+2) \subset \mathfrak{X} \times \mathbb{P}^{N}
$$

Let $\Xi \subset G(n+1-d, n+2) \times \check{\mathbb{P}}^{n+1}$ denote the tautological bundle, that is $\Xi=\left\{(L,[a]) \mid L \in G(n+1-d, n+2),[a] \in \mathbb{P} L \subset \check{\mathbb{P}}^{n+1}\right\}$, and consider the intersection

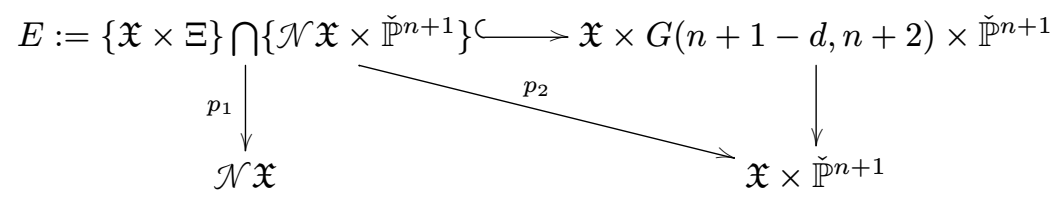

with the vertical morphism $p_{2}$ being the morphism induced by the projection onto $\mathfrak{X} \times \check{\mathbb{P}}^{n+1}$. We then have the following result.

Proposition 4.1. - Let $p_{2}: E \rightarrow \mathfrak{X} \times \check{\mathbb{P}}^{n+1}$ be as before. The set theoretical image $p_{2}(E)$ of the morphism $p_{2}$ coincides with the conormal space of $\mathfrak{X}$ in $\mathbb{C}^{n+2}$

$$
C(\mathfrak{X}) \subset \mathfrak{X} \times \check{\mathbb{P}}^{n+1}
$$

Moreover, the morphism $p_{1}: E \rightarrow \mathcal{N} \mathfrak{X}$ is a locally trivial fiber bundle over $\nu^{-1}\left(\mathfrak{X}^{0}\right) \subset \mathcal{N} \mathfrak{X}$ with fiber $\mathbb{P}^{n-d}$. 
Proof. - By definition, the conormal space of $\mathfrak{X}$ in $\mathbb{C}^{n+2}$ is an analytic space $C(\mathfrak{X}) \subset X \times \check{\mathbb{P}}^{n+1}$, together with a proper analytic map $\kappa: C(\mathfrak{X}) \rightarrow \mathfrak{X}$, where the fiber over a smooth point $x \in \mathfrak{X}^{0}$ is the set of tangent hyperplanes, that is the hyperplanes $H$ containing the direction of the tangent space $T_{x} \mathfrak{X}$. That is, if we define $E^{0}=\left\{(x, T,[a]) \in E \mid x \in \mathfrak{X}^{0}\right\}$, then by construction $E^{0}=p_{1}^{-1}\left(\nu^{-1}\left(\mathfrak{X}^{0}\right)\right)$, and $p_{2}\left(E^{0}\right)=C\left(\mathfrak{X}^{0}\right)$. Since the morphism $p_{2}$ is proper, in particular it is closed which finishes the proof.

In the same way we can construct the relative conormal space $C_{\varphi}(\mathfrak{X})$ as a subvariety of $\mathfrak{X} \times \check{\mathbb{P}}^{n}$ where $\check{\mathbb{P}}^{n}$ stands for the dual projective space of directions of hyperplanes of the hyperplane $\{t=0\} \subset \mathbb{C}^{n+1} \times \mathbb{C}$.

Proposition 4.2. - Let $Y \subset X$ be a smooth analytic subvariety of dimension $0 \leq s<d$, let $\varphi: \mathfrak{X} \rightarrow \mathbb{C}$ denote the specialization space of $X$ to its normal cone along $Y$, and let $\phi: \mathfrak{X} \rightarrow X \times \mathbb{C}$ denote the canonical map obtained from the construction in Lemma 2.2. Then there exist isomorphisms $\psi: C(\mathfrak{X} \backslash \mathfrak{X}(0)) \rightarrow$ $C(X) \times \mathbb{C}^{*} ; P: C(\mathfrak{X} \backslash \mathfrak{X}(0)) \rightarrow C_{\varphi}(\mathfrak{X} \backslash \mathfrak{X}(0))$; and $\psi_{\varphi}: C_{\varphi}(\mathfrak{X} \backslash \mathfrak{X}(0)) \rightarrow C(X) \times \mathbb{C}^{*}$ making the following diagram commutative:

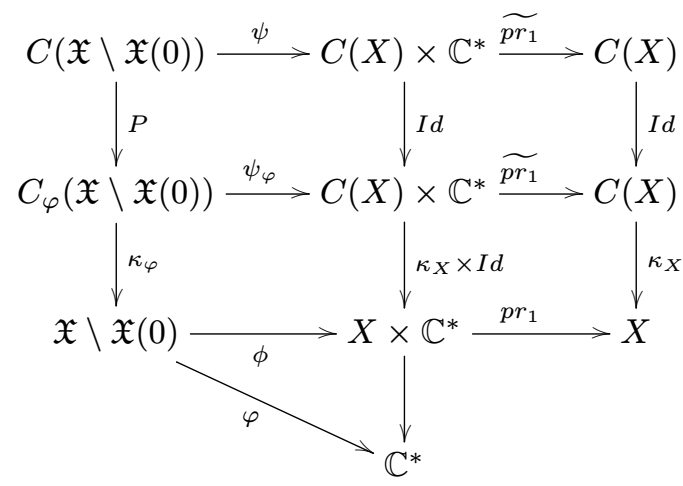

Proof. - We are working with a small enough representative of the germ $(X, 0) \subset\left(\mathbb{C}^{n+1}, 0\right)$ embedded in such a way that $Y \subset X$ is linear, this implies that we will have:

1. $C(X) \subset \mathbb{C}^{n+1} \times \check{\mathbb{P}}^{n}$

2. $\mathfrak{X} \subset \mathbb{C}^{n+1} \times \mathbb{C}$.

3. $C(\mathfrak{X}) \subset \mathbb{C}^{n+1} \times \mathbb{C} \times \check{\mathbb{P}}^{n+1}$

4. $C_{\varphi}(\mathfrak{X}) \subset \mathbb{C}^{n+1} \times \mathbb{C} \times \check{\mathbb{P}}^{n}$

We will actually work with the non-projectivized versions of the conormal spaces, that is with the spaces $T_{X}^{*}\left(\mathbb{C}^{n+1}\right), T_{\mathfrak{X}}^{*}\left(\mathbb{C}^{n+1} \times \mathbb{C}\right)$ and $T_{\mathfrak{X}}^{*}\left(\left(\mathbb{C}^{n+1} \times \mathbb{C}\right) / \mathbb{C}\right)$ respectively. Moreover, we will fix a coordinate system $\left(z_{0}, \ldots, z_{n-s}, y_{1}, \ldots, y_{s}, t, a_{0}, \ldots, a_{n-s}, c_{1}, \ldots, c_{s}, b\right)$ of $\mathbb{C}^{n+1} \times \mathbb{C}^{n} \check{\mathbb{C}}^{n+1} \times \check{C}$. 
By construction, the map $\phi: \mathfrak{X} \rightarrow X \times \mathbb{C}$ is an isomorphism when restricted to $\mathfrak{X} \backslash \mathfrak{X}(0)$ and has $X \times \mathbb{C}^{*}$ as its image. Actually, this alone implies that both the conormal space $C(\mathfrak{X} \backslash \mathfrak{X}(0))$ and the relative conormal space $C_{\varphi}(\mathfrak{X} \backslash \mathfrak{X}(0))$ are isomorphic to $C(X) \times \mathbb{C}^{*}$. However to verify that we have the commutative diagram we will specify these isomorphisms. Recall that the series

$$
F_{i}=t^{-m_{i}} f_{i}\left(t z_{0}, \ldots, t z_{n-s}, y_{1}, \ldots, y_{s}\right), i=1, \ldots, p
$$

define the specialization space $\mathfrak{X}$ in $\mathbb{C}^{n+1} \times \mathbb{C}$, where $m_{i}=\nu_{Y} f_{i}$.

Let $x=(z, y, t), t \neq 0$, be a smooth point of $\mathfrak{X}$, then it is a smooth point of $\mathfrak{X}(t)$, and $\phi(x)=(t z, y, t)$ is a smooth point of $X \times \mathbb{C}^{*}$; consequently $(t z, y)$ is a smooth point of $X$. Now, for any point $(x, a, c, b)$ in $\kappa_{\mathfrak{X}}^{-1}(x)$ we have that there exist constants $\lambda_{1}, \ldots, \lambda_{p}$ such that:

$$
a_{j}=\sum_{i=1}^{p} \lambda_{i} \frac{\partial F_{i}}{\partial z_{j}}(x)=\sum_{i=1}^{p} \lambda_{i} t^{-m_{i}+1} \frac{\partial f_{i}}{\partial z_{j}}(t z, y)
$$

$$
c_{j}=\sum_{i=1}^{p} \lambda_{i} \frac{\partial F_{i}}{\partial y_{j}}(x)=\sum_{i=1}^{p} \lambda_{i} t^{-m_{i}} \frac{\partial f_{i}}{\partial y_{j}}(t z, y)
$$

$$
b=\sum_{i=1}^{p} \lambda_{i} \frac{\partial F_{i}}{\partial t}(x)=\sum_{i=1}^{p} \lambda_{i}\left(\left(-m_{i}\right) t^{-m_{i}+1} f_{i}(t z, y)+t^{-m_{i}}\left(\sum_{k=0}^{n-s} z_{k} \frac{\partial f_{i}}{\partial z_{k}}(t z, y)\right)\right)
$$

$$
=\sum_{i=1}^{p} \lambda_{i}\left(t^{-m_{i}}\left(\sum_{k=0}^{n-s} z_{k} \frac{\partial f_{i}}{\partial z_{k}}(t z, y)\right)\right) \text {, because } f_{i}(t z, y)=0 \text { on } X \times \mathbb{C} .
$$

Analogously, for any point $(x, a, c)$ in $\kappa_{\varphi}^{-1}(x)$, there exist constants $\lambda_{1}, \ldots, \lambda_{p}$ such that, the coordinates $a_{j}$ and $c_{j}$ are given by the corresponding equations 2 and 3. This implies that the natural projection $P:(z, y, t, a, c, b) \mapsto(z, y, t, a, c)$ induces a surjective morphism to $C_{\varphi}(\mathfrak{X} \backslash \mathfrak{X}(0))$ when restricted to $C(\mathfrak{X} \backslash \mathfrak{X}(0))$. But, from 5 we can see that $t b=\sum_{k=0}^{n-s} z_{k} a_{k}$, so as long as $t \neq 0$ the $b$ coordinate is completely determined by the $a$ and $z$ coordinates which proves that the aforementioned map $P$ is an isomorphism.

On the other hand, for the corresponding point $x^{\prime}=(t z, y)$ of $X$, we have that for any point $\left(x^{\prime}, a, c\right)$ in $\kappa_{X}^{-1}\left(x^{\prime}\right)$ there exists constants $\alpha_{1}, \ldots, \alpha_{p}$ such 
that:

$$
\begin{aligned}
a_{j} & =\sum_{i=1}^{p} \alpha_{i} \frac{\partial f_{i}}{\partial z_{j}}(t z, y) \\
c_{j} & =\sum_{i=1}^{p} \alpha_{i} \frac{\partial f_{i}}{\partial y_{j}}(t z, y)
\end{aligned}
$$

This implies that if $t \neq 0$, the automorphism $\Upsilon: \mathbb{C}^{n+1} \times \mathbb{C} \times \check{\mathbb{C}}^{n+1} \circlearrowleft$ of the ambient space defined by:

$$
(z, y, t, a, c) \mapsto\left(t z_{0}, \ldots, t z_{n-s}, y_{1}, \ldots, y_{s}, t, a_{0}, \ldots, a_{n-s}, t c_{1}, \ldots, t c_{s}\right)
$$

induces a surjective map $\psi_{\varphi}: C_{\varphi}(\mathfrak{X} \backslash \mathfrak{X}(0)) \rightarrow C(X) \times \mathbb{C}^{*}$ simply by setting $\lambda_{i}=t^{m_{i}-1} \alpha_{i}$. Moreover, since the map $\Upsilon$ is biholomorphic in the open dense set $t \neq 0$, the map $\psi_{\varphi}$ is an isomorphism.

REMARK 4.3. - In regard to the previous diagrams, note that:

1. The map $\phi$ is defined on all of $\mathfrak{X}$, and the image of the special fiber $\mathfrak{X}(0)$ is just the origin in $X \times \mathbb{C}$. Note as well, that for a fixed $t \neq 0$, the morphism $p r_{1} \circ \phi \mid: \mathfrak{X}(t) \rightarrow X$ is an isomorphism.

2. The obstruction to the extension of $\psi$ to $C(\mathfrak{X})$ comes from the map $\check{\mathbb{P}}^{n+1} \rightarrow \check{\mathbb{P}}^{n}$, which is undefined at the point $[0: \cdots: 0: 1]$. This means that for any point $((\underline{z}, t),[\underline{a}: b])$ in $C(\mathfrak{X}) \cap\left(\mathfrak{X} \times\left\{\check{\mathbb{P}}^{n+1} \backslash[0: 1]\right\}\right)$, the hyperplane $[\underline{a}] \in \check{\mathbb{P}}^{n}$ is tangent to $X$ at the point $t \underline{z}=\left(t z_{0}, \ldots, t z_{n}\right)$. In particular, for $t=0$ the hyperplane $[\underline{a}]$ is tangent to $X$ at the origin.

\section{The Normal/Conormal diagram.}

Let $(Y, 0) \subset(X, 0)$ be a germ of a nonsingular analytic subvariety of dimension $s<d$ as before. The Whitney conditions of the pair $\left(X^{0}, Y\right)$ at 0 can be expressed in terms of the normal/conormal diagram of the pair $(X, Y, 0)$. We will choose an embedding $(X, 0) \subset\left(\mathbb{C}^{n+1}, 0\right)$ such that the germ $(Y, 0)$ is linear with coordinate system $\left(z_{0}, \ldots, z_{n-s}, y_{1}, \ldots, y_{s}\right)$.

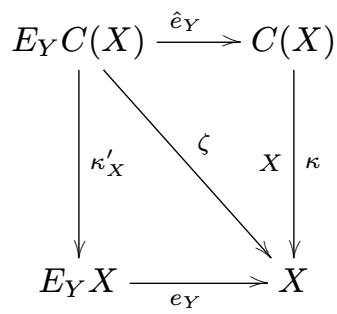

TOME $141-2013-\mathrm{N}^{\mathrm{O}} 2$ 
We will denote by $r:(X, 0) \longrightarrow(Y, 0)$ the retraction induced by the projection onto the $y$ coordinates.

Proposition 5.1. - Let $D$ denote the reduced divisor $\left|\zeta^{-1}(Y)\right| \subset E_{Y} C(X)$, then:

1. The pair $\left(X^{0}, Y\right)$ satisfies Whitney's condition a) at every point $y \in Y$ if and only if we have the set theoretical equality $|C(X) \cap C(Y)|=\left|\kappa_{X}^{-1}(Y)\right|$.

2. The pair $\left(X^{0}, Y\right)$ satisfies Whitney's condition a) at every point $y \in Y$ if and only if $D$ is contained in $Y \times \mathbb{P}^{n-s} \times \check{\mathbb{P}}^{n-s}$ where for every $y \in Y$, $\check{\mathbb{P}}^{n-s}$ denotes the space of hyperplanes containing $T_{y} Y$. In particular, they satisfy Whitney's condition a) at 0 if and only if $\zeta^{-1}(0) \subset\{0\} \times \mathbb{P}^{n-s} \times$ $\check{\mathbb{P}}^{n-s}$.

3. The pair $\left(X^{0}, Y\right)$ satisfies Whitney's condition $\left.b\right)$ at $y \in Y$ if and only if $\left|\zeta^{-1}(y)\right|$ is contained in the incidence variety $I \subset\{y\} \times \mathbb{P}^{n-s} \times \check{\mathbb{P}}^{n-s}$.

Proof. - Whitney conditions are defined in terms of limit of tangent spaces. However, once we have fixed an embedding $(X, 0) \subset\left(\mathbb{C}^{n+1}, 0\right)$, since a hyperplane $H$ is a limit of tangent hyperplanes if and only if it contains a limit of tangent spaces we can restate Whitney conditions:

-) The pair $\left(X^{0}, Y\right)_{0}$ satisfies Whitney condition a) at 0 if for any sequence of non-singular points $\left\{x_{i}\right\}_{i \in \mathbb{N}} \subset X^{0}$ tending to 0 , and any sequence $\left\{H_{i}\right\}_{i \in \mathbb{N}}$ where $H_{i}$ is a tangent hyperplane to $X$ at the point $x_{i}$ we have the inclusion

$$
T_{0} Y \subset \lim _{i \rightarrow \infty} H_{i}
$$

-) The pair $\left(X^{0}, Y\right)$ satisfies Whitney condition b) at $y \in Y$ if for any sequence of non-singular points $\left\{x_{i}\right\}_{i \in \mathbb{N}} \subset X^{0}$ tending to $y$, and any sequence $\left\{H_{i}\right\}_{\in \mathbb{N}}$ where $H_{i}$ is a tangent hyperplane to $X$ at the point $x_{i}$ we have the inclusion

$$
\lim _{i \rightarrow \infty}\left[x_{i} r\left(x_{i}\right)\right] \subset \lim _{i \rightarrow \infty} H_{i}
$$

With this in mind 1) is now only an observation. Note that we always have the inclusion $|C(X) \cap C(Y)| \subset\left|\kappa^{-1}(Y)\right|$. On the other hand, the inclusion $\left|\kappa^{-1}(Y)\right| \subset|C(Y)|$ means that for every $y \in Y$ every limit of tangent hyperplanes to $X$ at $y, H \in \kappa_{X}^{-1}(y)$, is also a tangent hyperplane to $Y$ at $y$, that is $T_{y} Y \subset H$.

For 2), with the coordinate system we have fixed we have the blowup $E_{Y} X$ as a subspace of $X \times \mathbb{P}^{n-s}$, and the conormal space $C(Y)$ equal to $Y \times \check{\mathbb{P}}^{n-s}$ where $\check{\mathbb{P}}^{n-s}$ corresponds to the projective dual of $\mathbb{P} Y$, that is the algebraic set defined by $c_{1}=\cdots=c_{s}=0$. Then, from 1) satisfying condition a) is equivalent to the inclusion $\left|\kappa_{X}^{-1}(Y)\right| \subset Y \times \check{\mathbb{P}}^{n-s}$ which by construction of the normal conormal diagram is equivalent to the inclusion $\left|\zeta^{-1}(Y)\right| \subset Y \times \mathbb{P}^{n-s} \times \check{\mathbb{P}}^{n-s}$. 
To prove 3), with the coordinate system we have fixed, we have the natural retraction $r: \mathbb{C}^{n+1} \rightarrow Y$ sending $(\underline{z}, \underline{y}) \rightarrow y$ which at the same time is used to build the underlying set of the blowup of $X$ along $Y, E_{Y} X$. So, from the construction of $E_{Y} C(X)$ as a subspace of the fiber product, we have to take the closure of the set of points of this space of the form $(\underline{z}, \underline{y}, l, H)$ where $(\underline{z}, \underline{y})$ is a point in $X^{0} \backslash Y, l \in \mathbb{P}^{n-s}$ is the line defined by $[(\underline{z}, \bar{y})-r(\underline{z}, y)]$ and $\bar{H}$ is a tangent hyperplane to $X$ at the point $(\underline{z}, y)$. Then, a point in the divisor $D=\zeta^{-1}(Y)$ is a point $(0, y, l, H)$, where $(0, y)$ is a point in $Y$, and $l$ and $H$ are a line and a hyperplane obtained in the way described in the definition of condition b) above. Finally the inclusion $l \subset H$ is just what it means that the pair $(l, H)$ is in the incidence variety $I \subset\{y\} \times \mathbb{P}^{n-s} \times \check{\mathbb{P}}^{n-s}$, which finishes the proof.

\section{Whitney's conditions.}

Let $(X, 0) \subset\left(\mathbb{C}^{n+1}, 0\right)$ be a reduced germ of an analytic singularity of pure dimension $d$, and let $\varphi:(\mathfrak{X}, 0) \rightarrow(\mathbb{C}, 0)$ denote the specialization of $X$ to its tangent cone $C_{X, 0}$. Let $\mathfrak{X}^{0}$ denote the open set of smooth points of $\mathfrak{X}$, and let $Y$ denote the smooth subspace $0 \times \mathbb{C} \subset \mathfrak{X}$. Our aim is to study the equisingularity of $\mathfrak{X}$ along $Y$, that is, we want to determine whether it is possible to find a Whitney stratification of $\mathfrak{X}$ in which the t-axis $Y$ is a stratum.

The first step to find out if such a stratification is possible, is to verify that the pair $\left(\mathfrak{X}^{0}, Y\right)$ satisfies Whitney's conditions. Since $\mathfrak{X} \backslash \mathfrak{X}(0)$ is isomorphic to the product $X \times \mathbb{C}^{*}$, Whitney's conditions are automatically verified everywhere in $\{0\} \times \mathbb{C}$, with the possible exception of the origin. The following result tells us that in this particular case it is enough to check for Whitney's condition a).

Proposition 6.1. - If the pair $\left(\mathfrak{X}^{0}, Y\right)$ satisfies Whitney's condition a) at the origin, then it also satisfies Whitney's condition b) at the origin.

Before proving Proposition 6.1, we need the following lemma.

LEMma 6.2. - There exists a natural morphism $\omega: E_{Y} \mathfrak{X} \rightarrow E_{0} X$, making the following diagram commute:

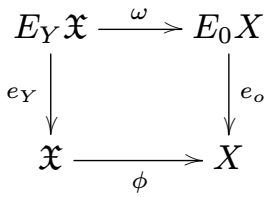

Moreover, when restricted to the exceptional divisor $e_{Y}^{-1}(Y)=\mathbb{P} C_{\mathfrak{X}, Y}$ it induces the natural map $\mathbb{P} C_{\mathfrak{X}, Y}=Y \times \mathbb{P} C_{X, 0} \rightarrow \mathbb{P} C_{X, 0}$.

TOME $141-2013-\mathrm{N}^{\mathrm{O}} 2$ 
Proof. - Algebraically, this results from the universal property of the blowup $E_{0} X$. We start with the diagram:

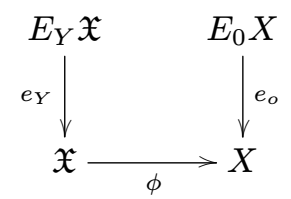

In this coordinate system, the maximal ideal $\mathfrak{m}$ of the analytic algebra $O_{X, 0}$ is generated by $\left\langle z_{0}, \ldots, z_{n}\right\rangle$. The map $\phi$, induces a morphism of analytic algebras $O_{X, 0} \rightarrow O_{\mathfrak{X}, 0}$ defined by $z_{i} \mapsto t z_{i}$. So we have to prove that the ideal $\left\langle t z_{0}, \ldots, t z_{n}\right\rangle \subset O_{\mathfrak{X}, 0}$ is locally invertible when pulled back to $E_{Y} \mathfrak{X}$. But as ideals we have the equality $\left\langle t z_{0}, \ldots, t z_{n}\right\rangle=\langle t\rangle \cdot\left\langle z_{0}, \ldots z_{n}\right\rangle$. And by definition of the blowup, the ideal $\left\langle z_{0}, \ldots, z_{n}\right\rangle \subset O_{\mathfrak{X}, 0}$ corresponding to $Y$ is locally invertible when pulled back to $E_{Y} \mathfrak{X}$. After multiplication by a invertible ideal, it will remain locally invertible. Note that, for the diagram to be commutative the morphism $\omega$ must map the point $(z, t),[z] \in E_{Y} \mathfrak{X} \backslash\left\{Y \times \mathbb{P}^{n}\right\} \subset \mathfrak{X} \times \mathbb{P}^{n}$ to the point $(t z),[z] \in E_{0} X \subset X \times \mathbb{P}^{n}$ and the result follows.

\section{REMARK 6.3. - Note that:}

1. For any point $y \in Y$, the tangent cone $C_{\mathfrak{X}, y}$ is isomorphic to $C_{X, 0} \times Y$, and the isomorphism is uniquely determined once we have chosen a set of coordinates. The reason is that for any $f(z)$ vanishing on $(X, 0)$, the function $F(z, t)=t^{-m} f(t z)=f_{m}+t f_{m+1}+t^{2} f_{m+2}+\ldots$, vanishes in $(\mathfrak{X}, 0)$ and so for any point $y=\left(\underline{0}, t_{0}\right)$ the initial form of $F\left(z, t+t_{0}\right)$ in $\mathbb{C}\left\{z_{0}, \ldots, z_{n}, t\right\}$ is equal to the initial form of $f$ at 0 . That is in ${ }_{\left(0, t_{0}\right)} F=$ $i n_{0} f$.

2. The projectivized normal cone $\mathbb{P} C_{\mathfrak{X}, Y}$ is isomorphic to $Y \times \mathbb{P} C_{X, 0}$. This can be seen from the equations used to define $\mathfrak{X}$ (Chapter 1, eq. 1), where the initial form of $F_{i}$ with respect to $Y$, is equal to the initial form of $f_{i}$ at the origin. That is $i n_{Y} F_{i}=i n_{0} f_{i}$.

Now we can proceed to the proof of 6.1 .

\section{Proof. - ( Proposition 6.1)}

We want to prove that the pair $\left(\mathfrak{X}^{0}, Y\right)$ satisfies Whitney's condition $\left.\mathrm{b}\right)$ at the origin. We are assuming that it already satisfies condition a), so in particular we have that $\zeta^{-1}(0)$ is contained in $\{0\} \times \mathbb{P}^{n} \times \check{\mathbb{P}}^{n}$. By Proposition 5.1 it suffices to prove that any point $(0, l, H) \in \zeta^{-1}(0)$ is contained in the incidence variety $I \subset\{0\} \times \mathbb{P}^{n} \times \check{\mathbb{P}}^{n}$. Consider the diagram: 


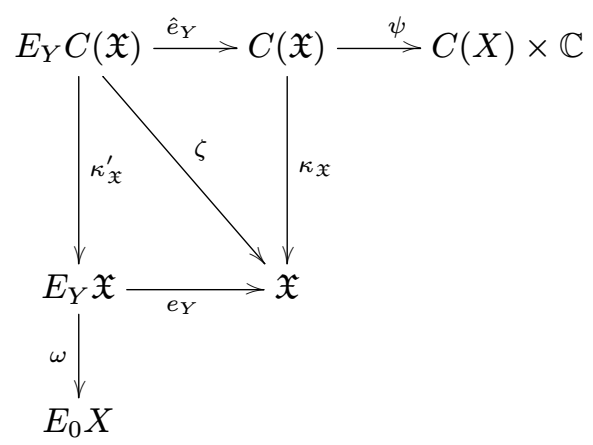

By construction, there is a sequence $\left(z_{m}, t_{m}, l_{m}, H_{m}\right)$ in $E_{Y} C(\mathfrak{X}) \hookrightarrow C(\mathfrak{X}) \times \mathfrak{X}$ $E_{Y} \mathfrak{X}$ tending to $(0, l, H)$ where $\left(z_{m}, t_{m}\right)$ is not in $Y$. Through $\kappa_{\mathfrak{X}}^{\prime}$, we obtain a sequence $\left(z_{m}, t_{m}, l_{m}\right)$ in $E_{Y} \mathfrak{X}$ tending to $(0, l)$, and through $\hat{e}_{Y}$ a sequence $\left(z_{m}, t_{m}, H_{m}\right)$ tending to $(0, H)$ in $C(\mathfrak{X})$.

Now, using the notation of Proposition 4.2, through the map $\psi$ we obtain the sequence $\left(t_{m} z_{m}, \widetilde{H}_{m}\right)$ and since by hypothesis we have $b=0$, then by Remark 4.3-2 both the sequence and its limit $(0, \widetilde{H})$ are in $C(X)$. Note that if $H$ has coordinates $\left[a_{0}: \cdots: a_{n}: 0\right]$, then $\widetilde{H}=\left[a_{0}: \cdots: a_{n}\right] \in \check{\mathbb{P}}^{n}$. On the other hand, by Lemma 6.2 we have that both the sequence $\left(t_{m} z_{m}, l_{m}\right)$ obtained through the map $\omega$ and its limit $(0, l)$ are in $E_{0} X$. Finally, Whitney's lemma ([17, Thm. 22.1, p. 547] or [14, Thm. 1.1.1]) tells us that in this situation we have that $l \subset \widetilde{H}$ and so the point $(0, l, H)$ is in the incidence variety.

If the sequence $\left(z_{m}, t_{m}, l_{m}, H_{m}\right)$ in $E_{Y} C(\mathfrak{X})$ is contained in the special fiber, that is $t_{m}=0$ for all $m$, then either the point $\left(z_{m}, 0\right)$ is a smooth point of $\mathfrak{X}$ and so the line $l_{m}=\left[z_{m}: 0\right]$ is contained in every tangent hyperplane $H_{m}$, or it is a singular point of $\mathfrak{X}$ and by constructing a sequence of smooth points in $\mathfrak{X} \backslash \mathfrak{X}(0)$ tending to it and using the maps $\psi$ and $\omega$ as we did before we prove that the line $l_{m}$ is contained in $H_{m}$. In any case, what we have is that for any point in the sequence $\left(z_{m}, 0, l_{m}, H_{m}\right)$ we already have the inclusion $l_{m} \subset H_{m}$ and so the limit $(0, l, H)$ satisfies this condition as well.

The following result tells us that in order to have $Y$ be a stratum in a Whitney stratification of $\mathfrak{X}$, the condition of $(X, 0)$ not having exceptional cones is necessary.

LEMMA 6.4. - Let $(X, 0) \subset\left(\mathbb{C}^{n+1}, 0\right)$ be a reduced germ of an analytic singularity of pure dimension $d$, and let $\varphi:(\mathfrak{X}, 0) \rightarrow(\mathbb{C}, 0)$ denote the specialization of $X$ to its tangent cone $C_{X, 0}$. Let $\mathfrak{X}^{0}$ denote the open set of smooth points of $\mathfrak{X}$, and let $Y$ denote the smooth subspace $0 \times \mathbb{C} \subset \mathfrak{X}$. If the tangent cone $C_{X, 0}$ 
is reduced and the pair $\left(\mathfrak{X}^{0}, Y\right)$ satisfies Whitney's condition a) then the germ $(X, 0)$ does not have exceptional cones.

Proof. - First of all, by hypothesis the pair $\left(\mathfrak{X}^{0}, Y\right)$ satisfies Whitney's condition a), so by Proposition 6.1 it also satisfies Whitney's condition b). Recall that the aureole of $(\mathfrak{X}, 0)$ along $Y$ is a collection $\left\{V_{\alpha}\right\}$ of subcones of the normal cone $C_{\mathfrak{X}, Y}$ whose projective duals determine the set of limits of tangent hyperplanes to $\mathfrak{X}$ at the points of $Y$ in the case that the pair $\left(\mathfrak{X}^{0}, Y\right)$ satisfies Whitney conditions a) and b) at every point of $Y$ (See [16, Thm. 2.1.1, Coro 2.1.2 p. 559-561] ). Among the $V_{\alpha}$ there are the irreducible components of $\left|C_{\mathfrak{X}, Y}\right|$. Moreover:

1. By Remark 6.3 we have that $C_{\mathfrak{X}, Y}=Y \times C_{X, 0}$ so its irreducible components are of the form $Y \times \widetilde{V}_{\beta}$ where $\widetilde{V}_{\beta}$ is an irreducible component of $\left|C_{X, 0}\right|$.

2. For each $\alpha$ the projection $V_{\alpha} \rightarrow Y$ is surjective and all the fibers are of the same dimension. (See [16][Proposition 2.2.4.2, p. 570])

3. The hyperplane $H=[0: 0: \cdots: 1] \in \check{\mathbb{P}}^{n+1}$ is transversal to $(\mathfrak{X}, 0)$ by hypothesis, and so by [16, Thm. 2.3.2, p. 572] the collection $\left\{V_{\alpha} \cap H\right\}$ is the aureole of $\mathfrak{X} \cap H$ along $Y \cap H$.

Notice that $(\mathfrak{X} \cap H, Y \cap H)$ is equal to $(\mathfrak{X}(0), 0)$, which is isomorphic to the tangent cone $\left(C_{X, 0}, 0\right)$ and therefore does not have exceptional cones. This means that for each $\alpha$ either $V_{\alpha} \cap H$ is an irreducible component of $C_{X, 0}$ or it is empty. But the intersection can't be empty because the projections $V_{\alpha} \rightarrow Y$ are surjective. Finally since all the fibers of the projection are of the same dimension then the $V_{\alpha}$ 's are only the irreducible components of $C_{\mathfrak{X}, Y}$. But this means, that if we define the affine hyperplane $H_{t}$ as the hyperplane with the same direction as $H$ and passing through the point $y=(0, t) \in Y$ for $t$ small enough; $H_{t}$ is transversal to $(\mathfrak{X}, y)$ and so we have again that the collection $\left\{V_{\alpha} \cap H_{t}\right\}$ is the aureole of $\mathfrak{X} \cap H_{t}$ along $Y \cap H_{t}$, that is the aureole of $(X, 0)$, so it does not have exceptional cones.

We can now use Lemma 6.4 to prove that the Whitney conditions of the pair $\left(\mathfrak{X}^{0}, Y\right)$ imply that the germ $(\mathfrak{X}, 0)$ does not have exceptional cones.

Proposition 6.5. - Let $(X, 0) \subset\left(\mathbb{C}^{n+1}, 0\right)$ be a reduced germ of an analytic singularity of pure dimension $d$, and let $\varphi:(\mathfrak{X}, 0) \rightarrow(\mathbb{C}, 0)$ denote the specialization of $X$ to its tangent cone $C_{X, 0}$. Let $\mathfrak{X}^{0}$ denote the open set of smooth points of $\mathfrak{X}$, and let $Y$ denote the smooth subspace $0 \times \mathbb{C} \subset \mathfrak{X}$.

1. If the germ $(\mathfrak{X}, 0)$ does not have exceptional cones, then the pair $\left(\mathfrak{X}^{0}, Y\right)$ satisfies Whitney's condition a) at the origin. 
2. Moreover, if the tangent cone $C_{X, 0}$ is reduced and the pair $\left(\mathfrak{X}^{0}, Y\right)$ satisfies Whitney's condition a) at the origin then $(\mathfrak{X}, 0)$ does not have exceptional cones.

Proof. - Let us choose a representative of $(X, 0)$ in $\left(\mathbb{C}^{n+1}, 0\right)$, then $(\mathfrak{X}, 0) \subset$ $\left(\mathbb{C}^{n+2}, 0\right)$. Let $C(\mathfrak{X}) \subset \mathbb{C}^{n+2} \times \check{\mathbb{P}}^{n+1}$ denote the conormal space of $\mathfrak{X}$, and let us consider the following diagram:

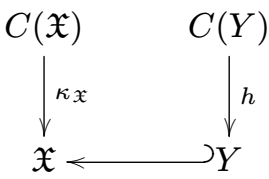

By Proposition 5.1, Whitney's condition a) at the origin is equivalent to the set theoretic inclusion

$$
\left|\kappa_{\mathfrak{X}}^{-1}(0)\right| \subset\left|h^{-1}(0)\right|
$$

Let $\left(\left(z_{0}, \ldots, z_{n}, t\right),\left[a_{0}: a_{1}: \ldots: a_{n}: b\right]\right)$ be the coordinates of $\mathbb{C}^{n+2} \times \check{\mathbb{P}}^{n+1}$ as before. Now, since $Y$ is the $t$ axis, the conormal space $C(Y)$ is defined by the equations $z_{0}=\cdots=z_{n}=b=0$, and for $h^{-1}(0)$ we just add the equation $t=0$.

1) By hypothesis $(\mathfrak{X}, 0)$ does not have exceptional cones, which means that $\left|\kappa_{\mathfrak{X}}^{-1}(0)\right|$ is just the dual of the tangent cone $C_{\mathfrak{X}, 0}=C_{X, 0} \times \mathbb{C}$. In particular, every tangent hyperplane to $C_{\mathfrak{X}, 0}$ contains the $t$ axis, that is $b=0$, so is contained in $h^{-1}(0)$, and we have Whitney's condition a).

2) By Lemma 6.4 we know that $(X, 0)$ does not have exceptional cones. Since every point in $\kappa_{\mathfrak{X}}^{-1}(0)$, that is every tangent hyperplane to $\mathfrak{X}$ at the origin satisfies $b=0$, the Remark 4.3-2 tells us that the morphism $\left(\widetilde{p r_{1}} \circ \psi\right)$ : $C(\mathfrak{X} \backslash \mathfrak{X}(0)) \rightarrow C(X)$ of Proposition 4.2, sending $(z, t),[a: b] \rightarrow(t z),[a]$ can be extended to $C(\mathfrak{X})$. In particular the point, $(0),[a]$ is in $\kappa_{X}^{-1}(0) \subset C(X)$, and since $(X, 0)$ does not have exceptional cones, then $[a]$ is in the dual of the tangent cone $C_{X, 0}$, which implies that $\kappa_{\mathfrak{X}}^{-1}(0)$ is just the dual of the tangent cone $C_{\mathfrak{X}, 0}$, and $(\mathfrak{X}, 0)$ does not have exceptional cones.

We will study Whitney's condition a) by deriving a characterization specific to our situation from the characterization given first by Teissier in [13] in the case of isolated hypersurface singularities and subsequently generalized by Gaffney in [2] in terms of integral dependence of modules.

\section{Limits of tangents spaces and integral closure of modules}

There are several equivalent definitions of integral closure for modules. In our case, it is simpler to work with the following definition, as stated in [3, Section 3, p. 555].

TOME $141-2013-\mathrm{N}^{\mathrm{O}} 2$ 
Definition 7.1. - Let $O_{\mathfrak{X}}^{p}$ be a free module of rank $p \geq 1$. Let $M$ be a coherent submodule of $O_{\mathfrak{X}}^{p}$ and $h \in O_{\mathfrak{X}}^{p}$. Given a map of germs $\phi:(\mathbb{C}, 0) \rightarrow(\mathfrak{X}, 0)$, denote by $h \circ \phi$ the induced section of $O_{1}^{p}$, and by $M \circ \phi$ the induced submodule. Call $h$ integrally dependent (resp. strictly dependent) on $M$ at 0 if, for every $\phi$, the section $h \circ \phi \in O_{1}^{p}$ belongs to the submodule $M \circ \phi$ of $O_{1}^{p}$ (resp. to the submodule $\left.\mathfrak{m}_{1}(M \circ \phi)\right)$, where $\mathfrak{m}_{1}$ is the maximal ideal of $O_{1}=\mathbb{C}\{\tau\}$. The submodule of $O_{\mathfrak{X}}^{p}$ generated by all such $h$ will be denoted by $\bar{M}$, respectively by $M^{\dagger}$.

Moreover, we say that a submodule $N \subset M$ is a reduction of $M$ if $\bar{N}=\bar{M}$.

If the germ $(\mathfrak{X}, 0)$ is not irreducible, for every irreducible component $\mathfrak{X}_{i}$ of $\mathfrak{X}$ the module $M$ induces a submodule $M_{\mathfrak{X}_{i}}$ of $O_{\mathfrak{X}_{i}}^{p}$ via the morphism of analytic algebras $O_{\mathfrak{X}, 0} \rightarrow O_{\mathfrak{X}_{i}, 0}$, and the same goes for a section $h$ of $O_{\mathfrak{X}}^{p}$. A simple calculation then shows:

LEMMA 7.2. - Let $(\mathfrak{X}, 0)=\bigcup_{i=1}^{r}\left(\mathfrak{X}_{i}, 0\right)$ be the irreducible decomposition of the germ. The section $h$ is integrally dependent (respectively strictly dependent) on $M$ at 0 if and only if for every irreducible component $\mathfrak{X}_{i}$ the induced section $h_{i}$ is integrally dependent (respectively strictly dependent) on $M_{\mathfrak{X}_{i}}$ at 0.

We will state the main results we will be using. Let $M$ be a coherent submodule of $O_{\mathfrak{X}}^{p}$ as before, and let $[M]$ be a matrix of generators of $M$ for a small enough neighborhood of the origin in $(\mathfrak{X}, 0)$, that is the matrix describing the morphism $\mu$ of:

$$
O_{\mathfrak{X}}^{q} \stackrel{\mu}{\longrightarrow} O_{\mathfrak{X}}^{p} \longrightarrow O_{\mathfrak{X}}^{p} / M \longrightarrow 0
$$

Let $J_{k}(M)$ denote the ideal of $O_{\mathfrak{X}}$ generated by the $k \times k$ minors of $[M]$. This is the same as the $(p-k)$-th Fitting ideal of $O_{\mathfrak{X}}^{p} / M$ and so is independent of the choice of generators of $M$. If $h \in O_{\mathfrak{X}}^{p}$, let $(h, M)$ denote the submodule of $O_{\mathfrak{X}}^{p}$ generated by $h$ and $M$.

Proposition 7.3. - [1, Prop 1.7, p. 304], and [2, Prop 1.5, p. 57]

Suppose $M$ is a submodule of $O_{\mathfrak{X}}^{p}, h \in O_{\mathfrak{X}}^{p}$ and the rank of $(h, M)$ is $k$ on each irreducible component of $(\mathfrak{X}, 0)$. Then $h$ is integrally dependent (resp. strictly dependent) on $M$ at 0 if and only if each minor in $J_{k}(h, M)$ which depends on $h$ is integrally dependent (resp. strictly dependent) on $J_{k}(M)$.

Lemma 7.4. - [3, Lemma 3.3, p. 557] For a section $h$ of $O_{\mathfrak{X}}^{p}$ to be integrally dependent, respectively strictly dependent, on $M$ at 0 , it is necessary that for all maps:

$$
\begin{aligned}
\phi:(\mathbb{C}, 0) & \rightarrow(\mathfrak{X}, 0) \\
\psi:(\mathbb{C}, 0) & \rightarrow\left(\operatorname{Hom}\left(\mathbb{C}^{p}, \mathbb{C}\right), \lambda\right), \quad \lambda \neq 0
\end{aligned}
$$


the function $\psi(h \circ \phi)$ on $\mathbb{C}$ belongs to the ideal $I_{\psi}(M \circ \phi)$ generated by applying $\psi(\tau)$ to the generators of $M \circ \phi$, respectively to the ideal $\mathfrak{m}_{1} I_{\psi}(M \circ \phi)$.

Conversely it is sufficient that this condition is satisfied for every $\phi:(\mathbb{C}, \mathbb{C} \backslash\{0\}, 0) \rightarrow(\mathfrak{X}, \mathfrak{X} \backslash W, 0)$, where $(W, 0) \subset(\mathfrak{X}, 0)$ is a proper analytic subset of $\mathfrak{X}$.

Corollary 7.5. - ( [1, Proposition 1.11, p. 306]) The section $h$ is integrally dependent on $M$ at 0 if and only if for each choice of generators $\left\{m_{i}\right\}$ of $M$ there exists a neighborhood $U$ of 0 in $\mathfrak{X}$, and a real constant $C$, such that for every section $\Psi: \mathfrak{X} \rightarrow \mathfrak{X} \times \check{\mathbb{P}}^{p-1}$ of the trivial bundle $\mathfrak{X} \times \check{\mathbb{P}}^{p-1}$ and every point $z \in U$ we have:

$$
|\Psi(z) \cdot h(z)| \leq C \sup _{i}\left|\Psi(z) \cdot m_{i}(z)\right|
$$

The previous results direct us to work with the space $\mathfrak{X} \times \check{\mathbb{C}}^{p}$, or even with the space $\mathfrak{X} \times \check{\mathbb{P}}^{p-1}$ since we ask that the image of $\psi$ does not contain the point 0 in $\check{C}^{p}$. These spaces can be seen respectively as the analytic spectrum (analytic proj) of the symmetric algebra of $O_{\mathfrak{X}}^{p}$, that is $O_{\mathfrak{X}}\left[u_{1}, \ldots, u_{p}\right]$. The section $h \in O_{\mathfrak{X}}^{p}$ and the submodule $M \subset O_{\mathfrak{X}}^{p}$ generate ideals in $O_{\mathfrak{X}}\left[u_{1}, \ldots, u_{p}\right]$ which we will denote by $\rho(h)$ and $\rho(M)$.

REMARK 7.6. - Recall that the embedding of $O_{\mathfrak{X}}^{p}$ in $O_{\mathfrak{X}}\left[u_{1}, \ldots, u_{p}\right]$ is in degree 1 , and is given by

$$
h=\left(\begin{array}{c}
h_{1} \\
h_{2} \\
\vdots \\
h_{p}
\end{array}\right) \mapsto \rho(h)=u_{1} h_{1}+\cdots+u_{p} h_{p}
$$

We will consider the normalized blowup of $\mathfrak{X} \times \check{\mathbb{P}}^{p-1}$ along the subspace $Z$ defined by the ideal $\rho(M) O_{\mathfrak{X}}\left[u_{1}, \ldots, u_{p}\right]$ which we will denote by

$$
\pi: \overline{E_{Z}(\mathfrak{X} \times \check{\mathbb{P}} p-1)} \rightarrow \mathfrak{X} \times \check{\mathbb{P}}^{p-1} \rightarrow \mathfrak{X}
$$

Its exceptional divisor will be denoted by $F$

Proposition 7.7. - [3, Prop. 3.5, p. 558] Let $h \in O_{\mathfrak{X}}^{p}$, and let $Y$ be a closed analytic subset of the image of $F$ in $\mathfrak{X}$. Then:

1. $h$ is integrally dependent on $M$ at 0 if and only if along each irreducible component of $F$, the ideal $\rho(h) \circ \pi$ vanishes to order at least the order of vanishing of $\rho(M) \circ \pi$.

2. $h$ is strictly dependent on $M$ at every $y \in Y$ if and only if along each component $V$ of $F$, the ideal $\rho(h) \circ \pi$ lies in the product $I(Y, V) \rho(M) \circ \pi$, where $I(Y, V)$ denotes the ideal of the reduced preimage of $Y$ in $V$.

TOME $141-2013-\mathrm{N}^{\mathrm{O}} 2$ 
From this point on we will assume that the germ $(\mathbf{X}, \mathbf{0})$ is irreducible.

Let $\left\langle F_{1}, \ldots, F_{p}\right\rangle \mathbb{C}\left\{z_{0}, \ldots, z_{n}, t\right\}$ be the ideal defining the germ $(\mathfrak{X}, 0)$ as before. In other words, $(\mathfrak{X}, 0)=\left(F^{-1}(0), 0\right)$ where $F=\left(F_{1}, \ldots, F_{p}\right):\left(\mathbb{C}^{n+1} \times\right.$ $\mathbb{C}, 0) \rightarrow\left(\mathbb{C}^{p}, 0\right)$. Let $c$ denote the codimension of $\mathfrak{X}$ in $\mathbb{C}^{n+1} \times \mathbb{C}$, and let $S$ denote the set of increasing sequences of $c$ positive integers less than $p+1$. For $\alpha \in S$ denote by $[D F]_{\alpha}$ the $c \times(n+2)$ submatrix of $[D F]$ formed by the $\left(\alpha_{1}, \ldots, \alpha_{c}\right)$ rows of $[D F]$. That is the Jacobian matrix, of the map $F_{\alpha}:=$ $\left(F_{\alpha_{1}}, \ldots F_{\alpha_{c}}\right): \mathbb{C}^{n+1} \times \mathbb{C} \rightarrow \mathbb{C}^{c}$.

DeFinition 7.8. - For $\alpha \in S$, define the $\alpha$-Jacobian module of $F$ as the submodule $J M(F)_{\alpha}$ of $O_{\mathfrak{X}}^{c}$ generated by the columns of the matrix $[D F]_{\alpha}$, that is:

$$
J M(F)_{\alpha}:=O_{\mathfrak{X}}\left(\begin{array}{c}
\frac{\partial F_{\alpha_{1}}}{\partial z_{0}} \\
\vdots \\
\frac{\partial F_{\alpha_{c}}}{\partial z_{0}}
\end{array}\right)+\cdots+O_{\mathfrak{X}}\left(\begin{array}{c}
\frac{\partial F_{\alpha_{1}}}{\partial z_{n}} \\
\vdots \\
\frac{\partial F_{\alpha_{c}}}{\partial z_{n}}
\end{array}\right)+O_{\mathfrak{X}}\left(\begin{array}{c}
\frac{\partial F_{\alpha_{1}}}{\partial t} \\
\vdots \\
\frac{\partial F_{\alpha_{c}}}{\partial t}
\end{array}\right) \subset O_{\mathfrak{X}}^{c}
$$

Let $v$ be a vector in $\mathbb{C}^{n+1} \times \mathbb{C}$, then by $\frac{\partial F_{\alpha}}{\partial v}$ we mean the directional derivative of $F_{\alpha}$ with respect to $v$. That is:

$$
\frac{\partial F_{\alpha}}{\partial v}:=[D F]_{\alpha}(v)
$$

In particular $\frac{\partial F_{\alpha}}{\partial v}$ is a linear combination of the columns of $F_{\alpha}$ and so it belongs to the $\alpha$-Jacobian module $J M(F)_{\alpha}$.

Definition 7.9. - Given an analytic map germ $g:\left(\mathbb{C}^{n+1} \times \mathbb{C}, 0\right) \rightarrow\left(\mathbb{C}^{l}, 0\right)$, and $\alpha \in S$, let $J M_{g}(F)_{\alpha}$ denote the submodule of $J M(F)_{\alpha}$ generated by the "partials" $\frac{\partial F_{\alpha}}{\partial v}$ for all vector fields $v$ on $\mathbb{C}^{n+1} \times \mathbb{C}$ tangent to the fibers of $g$, that is, for all $v$ that map to the 0 -field on $\mathbb{C}^{l}$. Call $J M_{g}(F)_{\alpha}$ the $\alpha$-Relative Jacobian Module with respect to $g$.

Note that if $H$ is a hyperplane in $\mathbb{C}^{n+1} \times \mathbb{C}$ defined by the kernel of the linear map $h: \mathbb{C}^{n+1} \times \mathbb{C} \rightarrow \mathbb{C}$, then $J M_{h}(F)_{\alpha}$ is the submodule of $J M(F)_{\alpha}$ generated by the partials $\frac{\partial F_{\alpha}}{\partial v}$ for all vectors $v \in H$.

REMARK 7.10. - 1. For every non-singular point $(z, t) \in \mathfrak{X}^{0}$, the matrix $[D F(z, t)]$ has rank $c:=n+1-d$, and so there exists an $\alpha \in S$ such that at least one of the maximal minors $(c \times c)$ of the matrix $[D F]_{\alpha}$ is not identically zero in $O_{\mathfrak{X}, 0}$.

2. For every point $(z, t)$ in the relative smooth locus $\mathfrak{X}_{\varphi}^{0}:=\bigcup \mathfrak{X}(t)^{0}$, the matrix $\left[D_{\varphi} F(z, t)\right]=\left[\frac{\partial F_{i}}{\partial z_{j}}\right]_{j=0 \ldots n}^{i=1 \ldots p}$ has rank $c:=n+1-d$, and so there exists a $\gamma \in S$ such that at least one of the maximal minors $(c \times c)$ of the matrix $\left[D_{\varphi} F\right]_{\gamma}$ is not identically zero in $O_{\mathfrak{X}, 0}$. 
The $\alpha$-relative Jacobian module, for an appropriately chosen $\alpha$, can be used to study the limits of tangent hyperplanes.

Proposition 7.11. - Let $\alpha \in S$ be as in Remark 7.10-1, and let $E_{Z}(\mathfrak{X} \times$ $\left.\check{\mathbb{P}}^{c-1}\right) \subset \mathfrak{X} \times \check{\mathbb{P}}^{c-1} \times \check{\mathbb{P}}^{n+1}$ be the blowup of $\mathfrak{X} \times \check{\mathbb{P}}^{c-1}$ along the subspace $Z$ defined by the ideal $\rho\left(J M(F)_{\alpha}\right) O_{\mathfrak{X}}\left[u_{1}, \ldots, u_{c}\right]$. Then, there exists a surjective map $\eta: E_{Z}\left(\mathfrak{X} \times \check{\mathbb{P}}^{c-1}\right) \rightarrow C(\mathfrak{X})$, making the following diagram commutative:

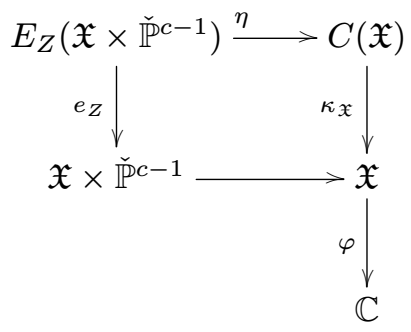

Proof. - Let $\alpha \in S$ be as in Remark 7.10. Since $\mathfrak{X}$ is irreducible, there exists an open dense set $\mathfrak{X}_{\alpha}^{0} \subset \mathfrak{X}^{0}$, where for any point $(z, t) \in \mathfrak{X}_{\alpha}^{0}$ the tangent space $T_{(z, t)} \mathfrak{X}$ is the kernel of the matrix $[D F]_{\alpha}$, that is, it is obtained as the intersection of the $c:=n+1-d$ hyperplanes $\left[\overrightarrow{d F_{\alpha_{j}}}(z, t)\right]$. Moreover, since $c$ is the codimension of $\mathfrak{X}$, any linear equation defining the tangent hyperplane $H=[a: b]$ to $\mathfrak{X}$ at $(z, t)$ is expressed as a unique linear combination of these $c$ hyperplanes $H=\left[\sum \beta_{j} \overrightarrow{d F_{\alpha_{j}}}(z, t)\right]$, that is, they form a base of the fiber $\kappa_{\mathfrak{X}}^{-1}(z, t)$ over $(z, t)$ in the conormal space $C(\mathfrak{X})$. So for any point $(z, t, u) \in$ $\mathfrak{X} \times \check{\mathbb{C}}^{c}$ with $(z, t) \in \mathfrak{X}_{\alpha}^{0}$ we have the map

$$
(z, t, u) \in \mathfrak{X} \times \check{\mathbb{C}}^{c} \mapsto(z, t),\left[\sum_{i=1}^{c} u_{i} \overrightarrow{d F_{\alpha_{i}}}(z, t)\right] \in C(\mathfrak{X}) \subset \mathfrak{X} \times \check{\mathbb{P}}^{n+1}
$$

Note that this map is invariant with respect to the homotheties of $\check{\mathbb{C}}^{c}$, so it defines a map $\mathfrak{X} \times \check{\mathbb{P}}^{c-1} \rightarrow \mathfrak{X} \times \check{\mathbb{P}}^{n+1}$.

On the other hand, from Definition 7.8 and Remark 7.6, we get that the ideal $\rho\left(J M(F)_{\alpha}\right)$ has the following system of homogeneous generators:

$$
\begin{aligned}
& \rho\left(J M(F)_{\alpha}\right) \\
& =\left\langle u_{1} \frac{\partial F_{\alpha_{1}}}{\partial z_{0}}+\cdots+u_{c} \frac{\partial F_{\alpha_{c}}}{\partial z_{0}}, \ldots, u_{1} \frac{\partial F_{\alpha_{1}}}{\partial t}+\cdots+u_{c} \frac{\partial F_{\alpha_{c}}}{\partial t}\right\rangle O_{\mathfrak{X}}\left[u_{1}, \ldots, u_{c}\right]
\end{aligned}
$$

and so a point $(z, t,[u]) \in \mathfrak{X} \times \check{\mathbb{P}}^{c-1}$ is in $Z$ if and only if

$$
u_{1} \overrightarrow{d F_{\alpha_{1}}}(z, t)+\cdots+u_{c} \overrightarrow{d F_{\alpha_{c}}}(z, t)=\overrightarrow{0}
$$

TOME $141-2013-\mathrm{N}^{\mathrm{O}} 2$ 
that is, $Z$ is the set of points where the previously stated map

$$
(z, t,[u]) \in \mathfrak{X} \times \check{\mathbb{P}}^{c-1} \mapsto(z, t),\left[\sum_{i=1}^{c} u_{i} \overrightarrow{d F_{\alpha_{i}}}(z, t)\right] \in C(\mathfrak{X}) \subset \mathfrak{X} \times \check{\mathbb{P}}^{n+1}
$$

is not defined. Thus, by blowing up the space $Z$ in this set of coordinates, we obtain the space $E_{Z}\left(\mathfrak{X} \times \check{\mathbb{P}}^{c-1}\right) \subset \mathfrak{X} \times \check{\mathbb{P}}^{c-1} \times \check{\mathbb{P}}^{n+1}$ upon which the morphism

$$
\eta: E_{Z}\left(\mathfrak{X} \times \check{\mathbb{P}}^{c-1}\right) \rightarrow C(\mathfrak{X})
$$

is defined by the restriction to $E_{Z}\left(\mathfrak{X} \times \check{\mathbb{P}}^{c-1}\right)$ of the projection $\mathfrak{X} \times \check{\mathbb{P}}^{c-1} \times$ $\check{\mathbb{P}}^{n+1} \rightarrow \mathfrak{X} \times \check{\mathbb{P}}^{n+1}$. Moreover, since for any point $(z, t) \in \mathfrak{X}_{\alpha}^{0}$ and tangent hyperplane $H \in \kappa_{\mathfrak{X}}^{-1}(z, t)$ there exists a unique $[u] \in \check{\mathbb{P}}^{c-1}$ such that the point $(z, t,[u]) \notin Z$ and the point $(z, t,[u], H) \in E_{Z}\left(\mathfrak{X} \times \check{\mathbb{P}}^{c-1}\right)$, then the morphism $\eta$ is surjective.

The proof of this proposition has the following result as an immediate corollary.

Corollary 7.12. - For each appropriately chosen $\alpha \in S$, the restriction of $\eta$ to $e_{Z}^{-1}\left(\mathfrak{X}_{\alpha}^{0}\right)$ is an isomorphism. In other words, the analytic spaces $\mathfrak{X}_{\alpha}^{0} \times \check{\mathbb{P}}^{c-1}$ and $\kappa_{\mathfrak{X}}^{-1}\left(\mathfrak{X}_{\alpha}^{0}\right)$ are isomorphic.

REMARK 7.13. - In the same spirit of the proof of the previous proposition, we can see that by choosing $a \gamma \in S$ as in Remark 7.10-2, the irreducibility of $\mathfrak{X}$ together with the constructive proof of 3.1 implies that the blowup of the ideal $J_{c}\left(J M_{\varphi}(F)_{\gamma}\right)$ generated by the maximal minors of $\left[D_{\varphi} F\right]_{\gamma}$ gives the relative Nash modification $\mathcal{N}_{\varphi} \mathfrak{X}$.

The link between limits of tangent hyperplanes and the integral closure is further explained in the following results.

Lemma 7.14. - ([2, Lemma 2.1, p. 58]) Let $(\mathfrak{X}, 0) \subset\left(\mathbb{C}^{n+1} \times \mathbb{C}, 0\right)$ be defined by $F^{-1}(0)$ as before, let $\alpha \in S$ be as in Remark $7.10-1$, and let $\mathfrak{X}_{\alpha}^{0}$ be the open dense set of smooth points of $\mathfrak{X}$ where the kernel of the matrix $[D F]_{\alpha}$ defines the tangent space $T_{(z, t)} \mathfrak{X}$. Then a hyperplane $H=\left[a_{0}: \cdots: a_{n}: b\right] \in \check{\mathbb{P}}^{n+1}$ is a limit of tangent hyperplanes to $(\mathfrak{X}, 0)$ if and only if there exists a pair of maps $\phi:(\mathbb{C}, \mathbb{C} \backslash 0,0) \rightarrow\left(\mathfrak{X}, \mathfrak{X}_{\alpha}^{0}, 0\right)$ and $\psi:(\mathbb{C}, 0) \rightarrow\left(\check{\mathbb{C}}^{c}, \lambda \neq 0\right)$ such that the point $(\phi(\tau), \psi(\tau)) \notin Z \subset \mathfrak{X} \times \check{\mathbb{C}}^{c}$ and for some $k$

$$
\left(a_{0}, \ldots, a_{n}, b\right)=\lim _{\tau \rightarrow 0} \frac{\psi(\tau) D F_{\alpha}(\phi(\tau))}{\tau^{k}}
$$


Proof. - The proof of this result is basically the same as that given in the reference just by noting the equivalence between a map

$$
\Theta:(\mathbb{C}, \mathbb{C} \backslash\{0\}, 0) \rightarrow\left(\mathfrak{X} \times \mathbb{C}^{c}, \mathfrak{X}_{\alpha}^{0} \times \mathbb{C}^{c},(0, \lambda)\right)
$$

and the pair of maps $\phi:(\mathbb{C}, \mathbb{C} \backslash\{0\}, 0) \rightarrow\left(\mathfrak{X}, \mathfrak{X}_{\alpha}^{0}, 0\right)$ and $\psi:(\mathbb{C}, 0) \rightarrow\left(\check{\mathbb{C}}^{c}, \lambda \neq\right.$ $0)$, and then using Propositon 7.11 and its corollary.

Corollary 7.15. - Let $\varphi:(\mathfrak{X}, 0) \rightarrow \mathbb{C}$ denote the specialization of $(X, 0)$ to its tangent cone $\left(C_{X, 0}, 0\right)$, and let $\alpha \in S$ be as in Remark \%.10-1. The hyperplane $\{t=0\}$ is not a limit of tangent hyperplanes to $\mathfrak{X}$ at $(z, t)$ if and only if $\frac{\partial F_{\alpha}}{\partial t} \in \overline{J M_{\varphi}(F)_{\alpha}}$ in $O_{\mathfrak{X},(z, t)}$.

Proof. - From Lemma 7.14, the hyperplane $\{t=0\}$ is a limit of tangent hyperplanes if and only if there exists a pair of maps $\phi:(\mathbb{C}, \mathbb{C} \backslash 0,0) \rightarrow\left(\mathfrak{X}, \mathfrak{X}_{\alpha}^{0}, 0\right)$ and $\psi:(\mathbb{C}, 0) \rightarrow\left(\check{\mathbb{C}}^{c}, \lambda \neq 0\right)$ such that the point $(\phi(\tau), \psi(\tau)) \notin Z \subset \mathfrak{X} \times \check{\mathbb{C}}^{c}$ and for some $\mathrm{k}$

$$
(0, \ldots, 0, b)=\lim _{\tau \rightarrow 0} \frac{\psi(\tau) D F_{\alpha}(\phi(\tau))}{\tau^{k}}
$$

But we can see that $\psi(\tau) D F_{\alpha}(\phi(\tau))$ is equal to

$$
\left(\rho\left(\frac{\partial F_{\alpha}}{\partial z_{0}}\right)(\phi(\tau), \psi(\tau)), \ldots, \rho\left(\frac{\partial F_{\alpha}}{\partial z_{n}}\right)(\phi(\tau), \psi(\tau)), \rho\left(\frac{\partial F_{\alpha}}{\partial t}\right)(\phi(\tau), \psi(\tau))\right)
$$

and so, if we denote by $\operatorname{ord}_{0} \gamma(\tau)$ the order of the series $\gamma(\tau)$ in $\mathbb{C}\{\tau\}$, the limit condition tells us that

$$
\operatorname{ord}_{0} \rho\left(\frac{\partial F_{\alpha}}{\partial t}\right)(\phi(\tau), \psi(\tau))<\operatorname{ord}_{0} \rho\left(\frac{\partial F_{\alpha}}{\partial z_{j}}\right)(\phi(\tau), \psi(\tau)), \text { for } j=0, \ldots, n
$$

This implies that for every $C \in \mathbb{R}$ there exists an $\epsilon \in \mathbb{R}$ such that for every $|\tau|<\epsilon$ we have that $\left|\rho\left(\frac{\partial F_{\alpha}}{\partial t}\right)(\phi(\tau), \psi(\tau))\right|>C\left|\rho\left(\frac{\partial F_{\alpha}}{\partial z_{j}}\right)(\phi(\tau), \psi(\tau))\right|$. Corollary 7.5 finishes the proof.

The equivalence statement of Whitney's condition a) in terms of integral closure and the Jacobian module given by Gaffney and Kleiman([2, Cor 2.4, p. 60 ] or [3, lemma 4.1, p. 560]) can now be refined in the irreducible case by using the $\alpha$-relative Jacobian module with basically the same proof.

\section{THEOREM 7.16. -}

Let $(X, 0) \subset\left(\mathbb{C}^{n+1}, 0\right)$ be an irreducible and reduced germ of an analytic singularity defined by an holomorphic map $f:\left(\mathbb{C}^{n+1}, 0\right) \rightarrow\left(\mathbb{C}^{p}, 0\right), X=f^{-1}(0)$. Let $(V, 0) \subset(X, 0)$ be a smooth subspace defined as the zero set of the analytic function $g:\left(\mathbb{C}^{n+1}, 0\right) \rightarrow\left(\mathbb{C}^{l}, 0\right)$, and let $\alpha \in S$ be as in Remark \%.10-1. Then the pair $\left(X^{0}, V\right)$ satisfies Whitney's condition a) at the origin if and only if the module $J M_{g}(f)_{\alpha}$ is contained in $J M(f)_{\alpha}^{\dagger}$. 
Corollary 7.17. - In the same setup of 7.16, let the smooth subspace $(V, 0) \subset(X, 0)$ be linear and defined by the projection $g:\left(\mathbb{C}^{n+1}, 0\right) \rightarrow\left(\mathbb{C}^{l}, 0\right)$ onto the first $l$ coordinates. If $h:\left(\mathbb{C}^{n+1}, 0\right) \rightarrow\left(\mathbb{C}^{n+1-l}, 0\right)$ denotes the retraction over $(V, 0)$, that is the projection onto the last $n+1-l$ coordinates, then the pair $\left(X^{0}, V\right)$ satisfies Whitney's condition a) at the origin if and only if the module $J M_{g}(f)_{\alpha}$ is contained in $J M_{h}(f)_{\alpha}^{\dagger}$.

Proof. - Recall that

$$
J M(f)_{\alpha}=\left\langle\left(\frac{\partial f_{\alpha}}{\partial z_{0}}\right), \cdots,\left(\frac{\partial f_{\alpha}}{\partial z_{n}}\right)\right\rangle O_{X}^{p}
$$

where $\left(\frac{\partial f_{\alpha}}{\partial z_{j}}\right)=\left(\begin{array}{c}\frac{\partial f_{\alpha_{1}}}{\partial z_{j}} \\ \vdots \\ \frac{\partial f_{\alpha_{c}}}{\partial z_{j}}\end{array}\right)$. Then, according to Definition 7.9 we have that:

$$
J M_{g}(f)_{\alpha}=\left\langle\left(\frac{\partial f_{\alpha}}{\partial z_{l}}\right), \cdots,\left(\frac{\partial f_{\alpha}}{\partial z_{n}}\right)\right\rangle O_{X}^{p}
$$

and

$$
J M_{h}(f)_{\alpha}=\left\langle\left(\frac{\partial f_{\alpha}}{\partial z_{0}}\right), \cdots,\left(\frac{\partial f_{\alpha}}{\partial z_{l-1}}\right)\right\rangle O_{X}^{p}
$$

Now, by definition, for a fixed map $(\phi, \psi):(\mathbb{C}, 0) \rightarrow\left(X \times \check{\mathbb{C}}^{p}, 0\right)$, with $(\phi(\tau),[\psi(\tau)]) \notin Z$ for $\tau \neq 0$, we have the ideal:

$$
\begin{aligned}
I_{\psi}\left(J M(f)_{\alpha} \circ \phi\right) & =\left\langle\psi(\tau)\left(\frac{\partial f_{\alpha}}{\partial z_{0}} \circ \phi\right), \cdots, \psi(\tau)\left(\frac{\partial f_{\alpha}}{\partial z_{n}} \circ \phi\right)\right\rangle \mathbb{C}\{\tau\} \\
& =\left\langle\tau^{r_{0}} w_{0}, \ldots, \tau^{r_{n}} w_{n}\right\rangle \mathbb{C}\{\tau\}, \text { with } w_{j} \in \mathbb{C}\{\tau\} \text { unit } \\
& =\left\langle\tau^{k}\right\rangle \mathbb{C}\{\tau\}
\end{aligned}
$$

But, by Theorem 7.16 we know that the pair $\left(X^{0}, V\right)$ satisfies Whitney's condition a) at the origin if and only if $J M_{g}(f)_{\alpha} \subset J M(f)_{\alpha}^{\dagger}$. That is, for $j=l, \ldots, n$ we have that

$$
\psi(\tau)\left(\frac{\partial f_{\alpha}}{\partial z_{j}} \circ \phi\right) \in \mathfrak{m}_{1} I_{\psi}\left(J M(f)_{\alpha} \circ \phi\right)=\left\langle\tau^{k+1}\right\rangle \mathbb{C}\{\tau\}
$$

so finally:

$$
\begin{aligned}
\left\langle\tau^{r_{0}} w_{0}, \ldots, \tau^{r_{n}} w_{n}\right\rangle \mathbb{C}\{\tau\} & =\left\langle\tau^{r_{0}} w_{0}, \ldots, \tau^{r_{l-1}} w_{l-1}\right\rangle \mathbb{C}\{\tau\} \\
& =I_{\psi}\left(J M_{h}(f)_{\alpha} \circ \phi\right)
\end{aligned}
$$

and the result follows. 
Corollary 7.18. - Let $\varphi:(\mathfrak{X}, 0) \rightarrow \mathbb{C}$ denote the specialization of $(X, 0)$ to its tangent cone $\left(C_{X, 0}, 0\right)$, and let $\alpha \in S$ be as in Remark 7.10-1. Then, the pair $\left(\mathfrak{X}^{0}, Y\right)$ satisfies Whitney's condition a) at the origin if and only if $\frac{\partial F_{\alpha}}{\partial t} \in J M_{\varphi}(F)_{\alpha}^{\dagger}$.

Proof. - For $(Y, 0) \subset(\mathfrak{X}, 0) \subset\left(\mathbb{C}^{n+1} \times \mathbb{C}, 0\right)$ we have that the projection

$$
\varphi:\left(\mathbb{C}^{n+1} \times \mathbb{C}, 0\right) \rightarrow(\mathbb{C}, 0)
$$

onto the last coordinate can be seen as the retraction over $(Y, 0)$. Moreover, the subspace $(Y, 0)$ is defined by the projection

$$
g:\left(\mathbb{C}^{n+1} \times \mathbb{C}, 0\right) \rightarrow\left(\mathbb{C}^{n+1}, 0\right)
$$

onto the first $n+1$ coordinates, so the module $J M_{g}(F)_{\alpha}=<\frac{\partial F_{\alpha}}{\partial t}>O_{\mathfrak{X}}^{p}$, and the result follows from 7.17.

Remark 7.19. - Proposition 7.11 gives us a relation between the blownup space $E_{Z}\left(\mathfrak{X} \times \check{\mathbb{P}}^{c-1}\right)$ and the limits of tangent hyperplanes for every point in a small enough neighborhood of the origin in $\mathfrak{X}$. Since it is this relation what gives the key to derive 7.14 to $\% .1 \%$, these results are also valid for every point in a small enough neighborhood of the origin in $\mathfrak{X}$; all we have to change is that the arcs $\phi:(\mathbb{C}, 0) \rightarrow\left(\mathfrak{X}^{0},(z, t)\right)$ arrive to the desired point. But more importantly, the characterization of Whitney's condition a) given in Corollary $\% .18$ is valid as stated for any sufficiently close point $y \in Y$.

\section{The Main Theorem}

Let $(X, 0)$ be a reduced germ of an analytic singularity of pure dimension $d$, with reduced tangent cone $C_{X, 0}$, and let $(X, 0)=\bigcup_{j=1}^{r}\left(X_{j}, 0\right)$ be its irreducible decomposition. By Lemma $2.5(\mathfrak{X}, 0)=\bigcup_{j=1}^{r}\left(\mathfrak{X}_{j}, 0\right)$ is the irreducible decomposition of the specialization space $\mathfrak{X}$, where $\left(\mathfrak{X}_{j}, 0\right)$ is the specialization space of the irreducible component $\left(X_{j}, 0\right)$ to its tangent cone $\left(C_{X_{j}, 0}, 0\right)$. Moreover, if the germ $(X, 0)$ does not have exceptional cones, by Lemma 8.1 the germs $\left(X_{j}, 0\right)$ don't have exceptional cones either. These two results allow us to restrict ourselves to the case where the germ $(X, 0)$ is irreducible which we have been treating.

Lemma 8.1. - The germ $(X, 0)$ does not have exceptional cones if and only if for each $i \in\{1, \ldots, r\}$ the germ $\left(X_{i}, 0\right)$ does not have exceptional cones.

Proof. - First of all, for a small enough representative of $X \subset \mathbb{C}^{n+1}$, we have the equality $C(X)=\bigcup C\left(X_{i}\right)$ where $C\left(X_{i}\right)$ denotes the conormal space of the embedding $X_{i} \subset \mathbb{C}^{n+1}$, and so the conormal map $\kappa_{X_{i}}$ is equal to the restriction of $\kappa_{X}$ to $C\left(X_{i}\right)$. Moreover, we know that the strict transform $\overline{e_{0}^{-1}\left(X_{i} \backslash\{0\}\right)}$ is 
equal to the blowup $E_{0} X_{i} \rightarrow X_{i}$, and since for every arc $\phi:(\mathbb{C}, 0) \rightarrow(X, 0)$ there exists a $j \in\{1, \ldots, r\}$ such that $\phi$ factorizes through $X_{j}$, we have the equality $\mathbb{P} C_{X, 0}=\bigcup \mathbb{P} C_{X_{i}, 0}$. All these imply that for each $i \in\{1, \ldots, r\}$ the normal conormal diagram

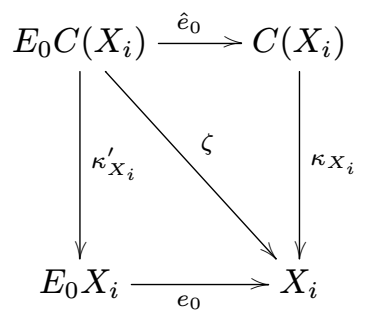

is canonically embedded in the normal conormal diagram of $X$ :

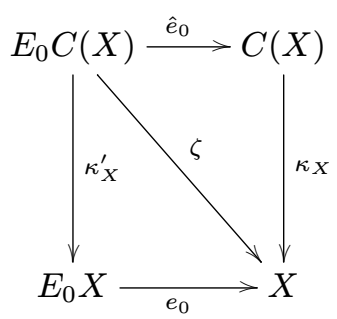

Now, the germ $(X, 0)$ does not have exceptional cones if and only if every irreducible component $W_{\alpha}$ of the fiber $\left|\kappa_{X}^{-1}(0)\right|=\bigcup\left|\kappa_{X_{i}}^{-1}(0)\right|$ is equal to the projective dual of an irreducible component $V_{\alpha}$ of the tangent cone $\mathbb{P} C_{X, 0}$, that is an irreducible component of one of the tangent cones $\mathbb{P} C_{X_{i}, 0}$. Finally, since for a reduced projective subvariety the double dual $\check{Y}$ is equal to $Y$, then two projective subvarieties $Y_{1}$ and $Y_{2}$ of $\mathbb{P}^{n}$ are different if and only if their duals are different $\check{Y}_{1} \neq \check{Y}_{2}$. This prevents the appearance of a possible exceptional cone of $X_{j}$ having the same dual as an irreducible component of $\mathbb{P} C_{X, 0}$ which finishes the proof.

As we have said before, the first step in our objective of constructing a Whitney stratification of $(\mathfrak{X}, 0)$ having the parameter axis $(Y, 0)$ as a stratum, is proving that the pair $\left(\mathfrak{X}^{\circ}, Y\right)$ satisfies Whitney conditions $\left.a\right)$ and $\left.b\right)$ at the origin. Since we are assuming $(\mathfrak{X}, 0)$ irreducible, what we have to prove, according to Corollary 7.18 is that for an $\boldsymbol{\alpha}$ chosen as in Remark 7.10-1, which we will fix from this point on, $\frac{\partial F_{\alpha}}{\partial t} \in J M_{\varphi}(F)_{\alpha}^{\dagger}$. So in terms of 7.3 , what we must prove (assuming we know that the rank of the Jacobian module is the codimension $c)$ is that every minor $M$ in $J_{c}\left(J M_{\alpha}(F)\right)$ depending on $\frac{\partial F_{\alpha}}{\partial t}$ satisfies $M \in J_{c}\left(J M_{\varphi}(F)_{\alpha}\right)^{\dagger}$. We will prove this using 7.7, and since we are working with ideals, it leads us to consider the normalized blowup of $\mathfrak{X}$ along the ideal 
$J_{c}\left(J M_{\varphi}(F)_{\alpha}\right)$. Moreover, by Remark 7.13, the blowup of $\mathfrak{X}$ along the ideal $J_{c}\left(J M_{\varphi}(F)_{\alpha}\right)$ gives the relative Nash modification $\nu_{\varphi}: \mathcal{N}_{\varphi} \mathfrak{X} \rightarrow \mathfrak{X}$.

Lemma 8.2. - The $\alpha$-Jacobian module $J M(F)_{\alpha}$ has rank $c$ on $(\mathfrak{X}, 0)$.

Proof. - By definition, the rank of a module over the integral domain $O_{\mathfrak{X}, 0}$ is the dimension as a vector space over the quotient field $Q\left(O_{\mathfrak{X}, 0}\right)$ of the vector space $Q\left(O_{\mathfrak{X}, 0}\right) \otimes J M(F)_{\alpha} O_{\mathfrak{X}, 0}$.

Consider the presentation

$$
O_{\mathfrak{X}, 0}^{r} \longrightarrow O_{\mathfrak{X}, 0}^{n+2} \stackrel{[D F]_{\alpha}}{\longrightarrow} J M(F)_{\alpha} O_{\mathfrak{X}, 0} \rightarrow 0
$$

where $[D F]_{\alpha}$ denotes the Jacobian matrix of the map $F_{\alpha}: \mathbb{C}^{n+2} \rightarrow \mathbb{C}^{c}$, which defines this map. By tensorizing this sequence by the field $Q\left(O_{\mathfrak{X}, 0}\right)$, we obtain the sequence

$$
Q\left(O_{\mathfrak{X}, 0}\right)^{r} \longrightarrow Q\left(O_{\mathfrak{X}, 0}\right)^{n+2} \stackrel{[D F]_{\alpha}}{\longrightarrow} Q\left(O_{\mathfrak{X}, 0}\right) \bigotimes J M_{\alpha}(F) O_{\mathfrak{X}, 0} \rightarrow 0
$$

where the map defined by the Jacobian matrix remains surjective. Remark that we now have that the rank of the module $J M_{\alpha}(F) O_{\mathfrak{X}, 0}$ is equal to the rank of the matrix $[D F]_{\alpha}$ when considering its entries as members of the quotient field $Q\left(O_{\mathfrak{X}, 0}\right)$.

Our choice of $\alpha$ guarantees the existence of a non zero $c \times c$ minor in $O_{\mathfrak{X}, 0}$. This implies that the ideal $J_{c}\left(J M(F)_{\alpha} O_{\mathfrak{X}, 0}\right)$ of $O_{\mathfrak{X}, 0}$ generated by all the $c \times c$ minors of the matrix $[D F]_{\alpha}$ is different from zero. Moreover since the matrix $[D F]_{\alpha}$ is of size $c \times(n+2)$, then the ideal $J_{c+1}\left(J M(F)_{\alpha} O_{\mathfrak{X}, 0}\right)$ is equal to the zero ideal. This remains true when considering the minors as elements of the quotient field $Q\left(O_{\mathfrak{X}, 0}\right)$, and so the rank of the matrix $[D F]_{\alpha}$ is equal to $c$ which finishes the proof.

We know that the pair $\left(\mathfrak{X}^{0}, Y\right)$ satisfies Whitney's conditions at every point $y$ of $Y$ with the possible exception of the origin, so we have by 7.19 that every minor $M$ in $J_{c}\left(J M(F)_{\alpha}\right)$ depending on $\frac{\partial F_{\alpha}}{\partial t}$ satisfies $M \in J_{c}\left(J M_{\varphi}(F)_{\alpha}\right)^{\dagger}$ in $O_{\mathfrak{X}, y}$ for all these points. What we are going to prove in Proposition 8.8 is that this condition carries over to the origin under the assumption that $(X, 0)$ does not have exceptional cones.

\section{REMARK 8.3. -}

1. The fact proven in Proposition 4.2, that the isomorphism between the conormal space $C(\mathfrak{X} \backslash \mathfrak{X}(0))$ and $C(X) \times \mathbb{C}^{*}$ is given by a natural projection implies that the vertical hyperplane $\{t=0\}:=[0: \cdots: 0: 1] \in \breve{\mathbb{P}}^{n+1}$ is not tangent to any point $(z, t) \in \mathfrak{X} \backslash \mathfrak{X}(0)$. This is equivalent, by Corollary 7.15, to $\frac{\partial F_{\alpha}}{\partial t} \in \overline{J M_{\varphi}(F)_{\alpha}}$ in $O_{\mathfrak{X},(z, t)}$ for every point $(z, t) \in \mathfrak{X} \backslash \mathfrak{X}(0)$.

томе $141-2013-\mathrm{N}^{\mathrm{O}} 2$ 
2. When $(\mathfrak{X}, 0)$ is a complete intersection, the center of the blowup defined by the ideal $J_{c}\left(J M_{\varphi}(F)\right)$ is set theoretically the relative singular locus of $\mathfrak{X}$. Moreover, since in this case, the tangent cone $\left(C_{X, 0}, 0\right)$ is a complete intersection, the equality $\frac{\partial F_{i}}{\partial z_{j}}(z, 0)=\frac{\partial f_{m_{i}}}{\partial z_{j}}(z)$ give us that the restriction of the ideal $J_{c}\left(J M_{\varphi}(F)\right)$ to the special fiber is equal to the Jacobian ideal $J_{C_{X, 0}}$ of the tangent cone $C_{X, 0}$ in $O_{C_{X, 0}}$. This implies that the strict transform of $\mathfrak{X}(0)$ with respect to this blowup is equal to the Nash modification $\mathcal{N} C_{X, 0}$ of the fiber.

3. Even though we are considering that $(X, 0)$ and as a result $(\mathfrak{X}, 0)$ are irreducible germs, this does not mean that the tangent cone $\left(C_{X, 0}\right)$ is irreducible. The problem with this is that the restriction of the ideal $J_{c}\left(J M_{\alpha}(F)\right)$ to the special fiber $\mathfrak{X}(0)$ may vanish in an irreducible component of the tangent cone $\left(C_{X, 0}, 0\right)$ and so its strict transform will no longer be equal to the Nash modification $\mathcal{N} C_{X, 0}$.

LEMmA 8.4. - For a reduced and irreducible germ $(X, 0)$ of an analytic singularity with reduced tangent cone $\left(C_{X, 0}, 0\right)$, there exists an ideal $I \subset O_{\mathfrak{X}, 0}$ such that:

1. The analytic subset $V(I) \subset \mathfrak{X}$ defined by $I$ contains the relative singular locus $\operatorname{Sing}_{\varphi} \mathfrak{X}:=\bigcup_{t}$ Sing $\mathfrak{X}(t)$.

2. The blowup of $\mathfrak{X}$ along $I$ is equal to the relative Nash modification of $\mathfrak{X}$, that is $E_{I} \mathfrak{X} \cong \mathcal{N} \varphi \mathfrak{X}$.

3. The blowup of the special fiber $\mathfrak{X}(0)$ along the ideal $I_{\mathfrak{X}(0), 0}$ defined by the restriction of $I$ to $\mathfrak{X}(0)$ is isomorphic to the Nash modification $\mathcal{N} C_{X, 0}$.

Proof. - Let $F:\left(\mathbb{C}^{n+1} \times \mathbb{C}, 0\right) \rightarrow\left(\mathbb{C}^{p}, 0\right)$ denote the germ of analytic map defined by the $p$ series $F_{1}, \ldots, F_{p} \in \mathbb{C}\left\{z_{0}, \ldots, z_{n}, t\right\}$, such that $(\mathfrak{X}, 0)=\left(F^{-1}(0), 0\right)$. Let $\left[D_{\varphi} F\right]$ denote the relative Jacobian matrix, and define the $p \times(n+1)$ matrix $A$ by setting the $t$ coordinate to 0 , that is $A=\left[D_{\varphi} F(z, 0)\right]$. By definition, $A$ is the Jacobian matrix of the map $g:\left(\mathbb{C}^{n+1}, 0\right) \rightarrow\left(\mathbb{C}^{p}, 0\right)$ defined by the homogeneous polynomials $g_{i}=F_{i}(z, 0)$ such that $\left(C_{X, 0}, 0\right)=\left(g^{-1}(0), 0\right)$. Let $c$ be the codimension of $\mathfrak{X}$ in $\mathbb{C}^{n+1} \times \mathbb{C}$, then $c$ is also the codimension of $C_{X, 0}$ in $\mathbb{C}^{n+1}$, and let $S\left(\right.$ resp. $\left.S^{\prime}\right)$ denote the set of increasing sequences of $c$-positive integers less than $p+1$ (resp. $n+2)$. For $\alpha=\left(\alpha_{1}, \ldots, \alpha_{c}\right) \in S$, and $\beta=\left(\beta_{1}, \ldots, \beta_{c}\right) \in S^{\prime}, g^{\alpha \beta}$ will denote the minor of $A$ obtained by considering the rows determined by $\alpha$ and the columns determined by $\beta$.

Let $C_{X, 0}=\bigcup_{j=1}^{l} V_{j}$ be the irreducible decomposition of the tangent cone. By the proof of 3.1 there exist $\alpha^{1}, \ldots, \alpha^{l}$ in $S$ and functions $h_{1}, \ldots, h_{l} \in O_{C_{X, 0}, 0}$, with $h_{i}=0$ on $\bigcup_{j \neq i} V_{j}$ and $h_{i} \neq 0$ on $V_{i}$, such that the blowup of $C_{X, 0}$ along the ideal $J=\left\langle\sigma_{\beta}:=\sum_{i=1}^{l} h_{i} g^{\alpha^{i}, \beta}, \beta \in S^{\prime}\right\rangle$ gives the Nash modification $\mathcal{N} C_{X, 0}$. 
Now, since for each $\alpha^{i}$ there is a non-zero minor of the matrix $[D g]_{\alpha^{i}}$, the corresponding minor of the matrix $\left[D_{\varphi} F\right]_{\alpha^{i}}$ is not identically zero. Since by hypothesis $\mathfrak{X}$ is irreducible then the proof of 3.1 tells us that this condition is enough to prove that the blowup of $\mathfrak{X}$ along the ideal $J_{c}\left(J M_{\varphi}(F)_{\alpha^{i}}\right)$ gives the relative Nash modification $\mathcal{N}_{\varphi} \mathfrak{X}$.

Let $F^{\alpha \beta}$ denote the minor of $\left[D_{\varphi} F\right]$ obtained by considering the rows determined by $\alpha$ and the columns determined by $\beta$, and define the ideal $I=$ $\left\langle\rho_{\beta}:=\sum_{i=1}^{l} h_{i} F^{\alpha^{i}, \beta}, \beta \in S^{\prime}\right\rangle$ where the $h_{i}$ 's are the same we used for the tangent cone. Now, by construction, the blowup of the special fiber $\mathfrak{X}(0)$ along the ideal $I O_{\mathfrak{X}(0), 0}$ is isomorphic to the Nash modification $\mathcal{N} C_{X, 0}$, and since for any point $(z, t)$ in the relative singular locus all the $c \times c$ minors of $\left[D_{\varphi} F\right]$ vanish, then we have the inclusion $\operatorname{Sing}_{\varphi} \mathfrak{X} \subset V(I)$. All that is left to prove, is that the blowup of $I$ gives $\mathcal{N}_{\varphi} \mathfrak{X}$.

Let $x=(z, t)$ be a point in the relative smooth locus of $\mathfrak{X}$ and $T_{x} \mathfrak{X}(t)^{0}=$ $\left[a_{0}: \ldots: a_{N}\right]$ denote the coordinates of the point of $\mathbb{P}^{N}$ corresponding to the direction of the tangent space to the fiber $\mathfrak{X}(t)$ at $x$ by the Plücker embedding of the grassmannian $G(d, n+1)$ in the projective space $\mathbb{P}^{N}$. If $(z, t)$ is sufficiently general then for each of the $\alpha^{i}$ 's we have:

$$
\left[F^{\alpha^{i}, \beta^{0}}: \cdots: F^{\alpha^{i}, \beta^{N}}\right]=\left[a_{0}: \ldots: a_{N}\right]
$$

where we have ordered the $\beta$ 's lexicographically. This means that there exist $\lambda_{1}, \ldots, \lambda_{l} \in \mathbb{C}$ such that for every $\alpha^{1}, \ldots, \alpha^{l}$ and $\beta^{k} \in S^{\prime}$ we have:

$$
F^{\alpha^{i}, \beta^{k}}=\lambda_{i} a_{k}
$$

which implies that for each $\beta^{k} \in S^{\prime}$ :

$$
\rho_{\beta^{k}}(x)=\sum_{i=1}^{l} h_{i} F^{\alpha^{i}, \beta^{k}}(x)=\sum_{i=1}^{l} h_{i} \lambda_{i} a_{k}=a_{k} \sum_{i=1}^{l} \lambda_{i} h_{i}
$$

and so $\left[\rho_{\beta}(x)\right]=[a]$ in $\mathbb{P}^{N}$. Finally, since the $\lambda^{\prime} s$ are non zero constants, the function $\sum_{i=1}^{l} \lambda_{i} h_{i}$ can not be identically zero. This implies that the equation $\left[\rho_{\beta}(x)\right]=[a]$ in $\mathbb{P}^{N}$ is true for every point $x$ in an open dense set $U \subset \mathfrak{X}$ which finishes the proof.

Proposition 8.5. - Let $\nu_{\varphi}: \mathcal{N}_{\varphi} \mathfrak{X} \rightarrow \mathfrak{X}$ be the relative Nash modification of $\varphi: \mathfrak{X} \rightarrow \mathbb{C}$. Let $Z \subset \mathfrak{X}$ be the subspace defined by the ideal $I$ of 8.4 , and let $D$ be the divisor defined by $I$ in $\mathcal{N}_{\varphi} \mathfrak{X}$, that is $D=\nu_{\varphi}^{-1}(Z)$. If the germ $(X, 0)$ does not have exceptional cones, then $\nu_{\varphi}^{-1}(Z \backslash \mathfrak{X}(0))$ is dense in $D$. That is, the exceptional divisor $D$ of $\mathcal{N}_{\varphi} \mathfrak{X}$ does not have vertical components over $\mathfrak{X}(0)$.

Proof. - We know that $\mathfrak{X}(0)$ is isomorphic to the tangent cone $C_{X, 0}$. Now, by ?? the strict transform of $\mathfrak{X}(0)$ in $\mathcal{N}_{\varphi}(\mathfrak{X})$ is isomorphic to the Nash modification 
$v: \mathcal{N} C_{X, 0} \rightarrow C_{X, 0}$. Moreover, by the definition of blowing up, $v^{-1}(Z \cap \mathfrak{X}(0))$ is a divisor (of dimension $d-1$ ).

Now, by ?? if $(z, 0, T) \in \nu_{\varphi}^{-1}(z, 0) \subset \mathcal{N}_{\varphi} \mathfrak{X}$ then the $d$-plane $T$ is via $\Gamma$ a limit of tangent spaces to $X$ at 0 , that is the point $(0, T) \in \nu^{-1}(0) \subset \mathcal{N} X$. But, since by hypothesis the germ $(X, 0)$ does not have exceptional cones, then $T$ is tangent to the tangent cone $C_{X, 0}$.

We want to prove that the total transform $\nu_{\varphi}^{-1}(\mathfrak{X}(0))$ coincides with the strict transform $\mathcal{N} C_{X, 0}$, that is, we need to prove that the point $(z, 0, T)$ is in $\mathcal{N} C_{X, 0}$. For this purpose all that is now left to prove is that $T$ is tangent to $C_{X, 0}$ at the point $p=(z)$.

Let $\delta:(\mathbb{C}, \mathbb{C} \backslash\{0\}, 0) \rightarrow\left(X, X^{0}, 0\right)$ be an arc such that its lift $\widetilde{\delta}$ to $\mathcal{N} X$ has the point $(0, T) \in \mathcal{N} X$ as endpoint.

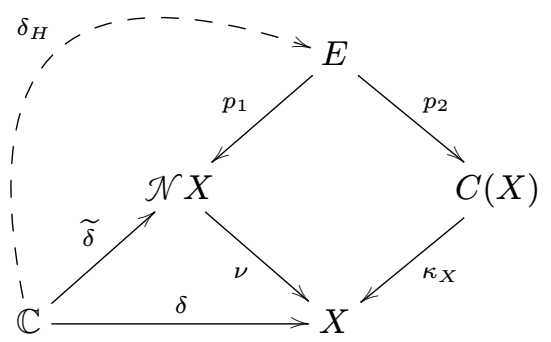

By construction $\delta(\mathbb{C} \backslash\{0\})$ is contained in the smooth locus $X^{\circ}$, and if we denote by $E^{\circ}$ the inverse image $p_{1}^{-1}\left(\nu^{-1}\left(X^{0}\right)\right)$, then by 4.1 the open subset $E^{\circ}$ is dense in $E$, and it defines a locally trivial fiber bundle over $\nu^{-1}\left(X^{\circ}\right)$. This implies that for any point $(0, T, H) \in p_{1}^{-1}(0, T)$ the arc $\widetilde{\delta}$ can be lifted to an $\operatorname{arc} \delta_{H}$ having the point $(0, T, H)$ as endpoint. So now we have transformed the problem into proving that any hyperplane $H \in \check{\mathbb{P}}^{n}$, such that $T \subset H$, is a tangent hyperplane to $C_{X, 0}$ at the point $p=z$.

Going back again to the diagram of 3.2 :

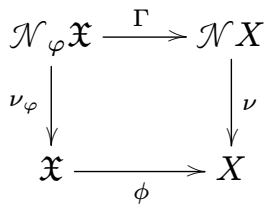

we have that for any sequence $\left\{\left(z_{m}, t_{m}\right)\right\}$ in the smooth part of $\mathfrak{X} \backslash \mathfrak{X}(0)$ tending to the point $(z, 0)$ in the special fiber $\mathfrak{X}(0)$, we have a corresponding sequence 
$\left\{\left(t_{m} z_{m}\right)\right\}$ tending to the origin in $X$. The final step of the proof is now a consequence of the projective duality obtained from the normal/conormal diagram:

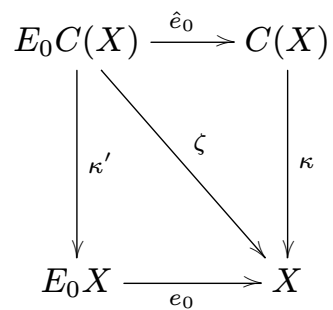

since the sequence $\left\{t_{m} z_{m}\right\} \subset X \backslash\{0\}$ tending to the origin gives us the sequence $\left\{\left(t_{m} z_{m}\right),\left[z_{m}\right]\right\}$ in $E_{0} X$, the blowing up of $X$ at 0 , which tends to the point $(0,[z])$ in the exceptional divisor $\mathbb{P} C_{X, 0}$. In the same way, we obtain the sequence $\left\{\left(t_{m} z_{m},\left[z_{m}\right], H_{m}\right)\right\}$ in $E_{0} C(X) \subset X \times \mathbb{P}^{n} \times \check{\mathbb{P}}^{n}$ tending to the point $(0,[z], H)$ in $G=\zeta^{-1}(0)$. Recall that if $|G|=\bigcup_{\alpha} G_{\alpha}$ is the irreducible decomposition of the reduced space $|G|$, then each $G_{\alpha}$ is the conormal space of an irreducible component of $\mathbb{P} C_{X, 0}$. To finish the proof, note that so far we have proved that $\nu_{\varphi}^{-1}(\mathfrak{X}(0))$ is just $\mathcal{N} C_{X, 0}$ and so $\nu_{\varphi}^{-1}(Z(0))$ is of dimension $d-1$, whereas an irreducible component of $D$ is of dimension $d$.

Corollary 8.6. - Let Sing $\mathfrak{X}(0)$ denote the singular locus of the special fiber, then the dimension of $\nu_{\varphi}^{-1}(\operatorname{Sing} \mathfrak{X}(0))$ is less or equal than $d-1$.

Proof. - By definition of the ideal $I$, the analytic subset Sing $\mathfrak{X}(0)$ is contained in the subspace $Z$ defined by $I$. Then we have the inclusion $\nu_{\varphi}^{-1}(\operatorname{Sing} \mathfrak{X}(0)) \subset$ $\nu_{\varphi}^{-1}(Z(0))$ and by Proposition 8.5 the dimension of $\nu_{\varphi}^{-1}(Z(0))$ is equal to $d-1$ which finishes the proof.

Note that the following result does not uses the irreducible hypothesis, and so is valid in a more general setting.

Lemma 8.7. - Let $Y$ denote the smooth subspace $0 \times \mathbb{C} \subset \mathfrak{X}$ as before, let $\nu: \mathcal{N} X \rightarrow X$ be the Nash modification of $X$, and let $\widetilde{\nu_{\varphi}}: \widetilde{\mathcal{N}_{\varphi} \mathfrak{X}} \rightarrow \mathfrak{X}$ be the normalized relative Nash modification of $\mathfrak{X}$. Then:

1. If the germ $(X, 0)$ does not have exceptional cones we have the settheoretical equality:

$$
\left|\nu_{\varphi}^{-1}(Y)\right|=\left|Y \times \nu^{-1}(0)\right|
$$

2. The set theoretical inverse image $\left|{\widetilde{\nu_{\varphi}}}^{-1}(Y \backslash\{0\})\right|$ is dense in $\left|{\widetilde{\nu_{\varphi}}}^{-1}(Y)\right|$.

TOME $141-2013-\mathrm{N}^{\circ} 2$ 
Proof. - From Proposition 3.2 we have the commutative diagram:

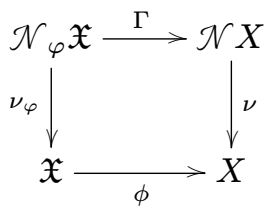

where $\phi$ and $\Gamma$ are surjective. The morphism $\phi$ is the restriction to $\mathfrak{X}$ of the map $\mathbb{C}^{n+1} \times \mathbb{C} \rightarrow \mathbb{C}^{n+1}$ defined by $\left(z_{0}, \ldots, z_{n}, t\right) \mapsto\left(t z_{0}, \ldots, t z_{n}\right)$ which is an isomorphism on $\mathbb{C}^{n+1} \times \mathbb{C}^{*}$. This implies in particular that the restriction of the differential $D \phi$ to the tangent space $T_{(z, t)} \mathfrak{X}(t)$ maps it isomorphically to $T_{(t z)} X$, where $(z, t)$ is a smooth point of the fiber $\mathfrak{X}(t)$ with $t \neq 0$. But the restriction of $D \phi$ to $T_{(z, t)} \mathfrak{X}(t)$ is $t$ times the identity Id, which implies that $\nu_{\varphi}^{-1}\left(Y \backslash\{(0,0)\}=Y \backslash\{(0,0)\} \times \nu^{-1}(0)\right)$ and as a consequence $\nu_{\varphi}^{-1}(0,0)$ contains $\nu^{-1}(0)$. Finally, from the proof of Proposition 8.5 we know that the fiber $\nu_{\varphi}^{-1}(\mathfrak{X}(0))$ is equal to the Nash modification of the tangent cone $C_{X, 0}$, so the fiber $\nu_{\varphi}^{-1}(0,0)$ is equal to the set of limits of tangent spaces to $C_{X, 0}$ which coincides with $\nu^{-1}(0)$ since the germ $(X, 0)$ does not have exceptional cones.

To prove 2), note that since $\nu_{\varphi}^{-1}(Y)$ has a product structure we already have that $\nu_{\varphi}^{-1}(Y \backslash\{0\})$ is dense in $\nu_{\varphi}^{-1}(Y)$, and so we need to study how the normalization $n: \widetilde{\mathcal{N}_{\varphi} \mathfrak{X}} \rightarrow \mathcal{N}_{\varphi} \mathfrak{X}$ affects this subspace. Let $(0,0, T) \in$ $\mathcal{N}_{\varphi} \mathfrak{X}$ be a point over the origin in $\mathfrak{X}$. Since by hypothesis $\mathfrak{X}$ is irreducible, the space $\mathcal{N}_{\varphi} \mathfrak{X}$ is also irreducible, however it may not be locally irreducible so the germ $\left(\mathcal{N}_{\varphi} \mathfrak{X},(0,0, T)\right)$ may have an irreducible decomposition of the form $\left(\mathcal{N}_{\varphi} \mathfrak{X},(0,0, T)\right)=\bigcup_{j}\left(W_{j},(0,0, T)\right)$. Now, by [7, Section 4.4], we have that the normalization map is finite, and over $\left(\mathcal{N}_{\varphi} \mathfrak{X},(0,0, T)\right)$ in the normalized space $\widetilde{\mathcal{N}_{\varphi} \mathfrak{X}}$ we have a multigerm $\bigsqcup_{j}\left(\widetilde{W_{j}}, p_{j}\right)$ such that:

1. The germ $\left(\widetilde{W_{j}}, p_{j}\right)$ is irreducible, and corresponds to the normalization of $\left(W_{j},(0,0, T)\right)$.

2. For every $j$ we have that $n^{-1}(0,0, T) \cap \widetilde{W_{j}}=\left\{p_{j}\right\}$.

This implies that if $\nu_{\varphi}\left(\nu_{\varphi}^{-1}(Y) \cap W_{j}\right)=Y$, then set-theoretically ${\widetilde{\nu_{\varphi}}}^{-1}(Y \backslash$ $\{0\}) \cap \widetilde{W_{j}}$ is dense in ${\widetilde{\nu_{\varphi}}}^{-1}(Y) \cap \widetilde{W_{j}}$, and so all we have to prove is that every $W_{j}$ satisfies this condition.

Since the open set of relative smooth points $\mathfrak{X}_{\varphi}^{0} \backslash \mathfrak{X}(0)$ is dense in $\mathfrak{X}$, then its preimage $\nu_{\varphi}^{-1}\left(\mathfrak{X}_{\varphi}^{0} \backslash \mathfrak{X}(0)\right)$ is dense in $\mathcal{N}_{\varphi} \mathfrak{X}$ and so it intersects every irreducible component $W_{j}$ in an open dense set $U_{j}$. This means that there exists an arc contained in $U_{j}$

$$
\begin{aligned}
\mu:(\mathbb{C}, \mathbb{C} \backslash\{0\}, 0) & \rightarrow\left(W_{j}, U_{j},(0,0, T)\right) \\
\tau & \mapsto(z(\tau), t(\tau), T(\tau))
\end{aligned}
$$


having $(0,0, T)$ as endpoint; moreover by composing it with $\nu_{\varphi}$ we get an $\operatorname{arc}$

$$
\widetilde{\mu}:(\mathbb{C}, \mathbb{C} \backslash\{0\}, 0) \rightarrow\left(\mathfrak{X}, \mathfrak{X}_{\varphi}^{0} \backslash \mathfrak{X}(0),(0,0)\right)
$$

contained in $\mathfrak{X}_{\varphi}^{0} \backslash \mathfrak{X}(0)$ having the origin as endpoint.

Let $\widetilde{\mu}=(z(\tau), t(\tau))$ and let $\alpha \in \mathbb{C}^{*}$, by Propositions 4.2 and 3.2 , this arc can be "verticalized" to an arc $\widetilde{\mu_{\alpha}}:(\mathbb{C}, \mathbb{C} \backslash\{0\}, 0) \rightarrow\left(\mathfrak{X}(\alpha), \mathfrak{X}^{0}(\alpha),(0, \alpha)\right)$ as follows:

$$
\begin{aligned}
(\mathbb{C}, \mathbb{C} \backslash\{0\}, 0) & \rightarrow\left(\mathfrak{X}, \mathfrak{X}_{\varphi}^{0} \backslash \mathfrak{X}(0),(0,0)\right) \longrightarrow\left(X, X^{0}, 0\right) \longrightarrow\left(\mathfrak{X}(\alpha), \mathfrak{X}(\alpha)^{0},(0, \alpha)\right) \\
\tau & \mapsto(z(\tau), t(\tau)) \longmapsto(t(\tau) z(\tau)) \longmapsto\left(\frac{t(\tau) z(\tau)}{\alpha}, \alpha\right)
\end{aligned}
$$

Since the canonical isomorphism between two fibers $\mathfrak{X}\left(\alpha_{1}\right)$ and $\mathfrak{X}\left(\alpha_{2}\right)$ used here is given by $\left(z, \alpha_{1}\right) \mapsto\left(\frac{\alpha_{1}}{\alpha_{2}} z, \alpha_{2}\right)$, for every smooth point the tangent map acts as $\frac{\alpha_{1}}{\alpha_{2}}$ times the identity on the embedded tangent space leaving it invariant. Now, since the arc is contained in the smooth locus $\mathfrak{X}^{0}(\alpha)$ it has a unique lift to an arc

$$
\mu_{\alpha}:(\mathbb{C}, \mathbb{C} \backslash\{0\}, 0) \rightarrow\left(\mathcal{N}_{\varphi} \mathfrak{X}, \nu_{\varphi}^{-1}\left(\mathfrak{X}_{\varphi}^{0}\right),(0, \alpha, T)\right)
$$

having as endpoint the point $(0, \alpha, T)$. Moreover for every $\tau_{0}$ close enough to the origin in $\mathbb{C}$ the point $\left(z\left(\tau_{0}\right), t\left(\tau_{0}\right), T\left(\tau_{0}\right)\right)$ is in $W_{j}$ and since the arc $\mu_{t\left(\tau_{0}\right)}$ passes through this point, then it is completely contained in $W_{j}$, in particular the endpoint $\left(0, t\left(\tau_{0}\right), T\right)$ is in $W_{j}$ which finishes the proof.

We are now in position to prove that $\frac{\partial F_{\alpha}}{\partial t}$ is strictly dependent on $J M_{\varphi}(F)_{\alpha}$ at 0 .

Proposition 8.8. - If the germ $(X, 0)$ does not have exceptional cones then every minor $M$ in $J_{c}\left(J M(F)_{\alpha}\right)$ depending on $\frac{\partial F_{\alpha}}{\partial t}$ satisfies $M \in J_{c}\left(J M_{\varphi}(F)_{\alpha}\right)^{\dagger}$ in $O_{X, 0}$.

Proof. - Let $M$ be a minor in $J_{c}\left(J M(F)_{\alpha}\right)$ that depends on $\frac{\partial F_{\alpha}}{\partial t}$, and let $W \subset \mathfrak{X}$ be the subspace defined by the ideal $J_{c}\left(J M_{\varphi}(F)_{\alpha}\right)$. Note that by definition, not only the $t$-axis $Y$, but the entire relative singular locus $\operatorname{Sing}_{\varphi} \mathfrak{X}$ is contained in $W$. Let $\widetilde{\nu_{\varphi}}: \widetilde{\mathcal{N}_{\varphi} \mathfrak{X}} \rightarrow \mathfrak{X}$ be the normalized blowup of $\mathfrak{X}$ along $J_{c}\left(J M_{\varphi}(F)_{\alpha}\right)$, and let $\bar{D}$ be its exceptional divisor. By considering a small enough neighborhood of the origin in $\mathfrak{X}$, or in other words a small enough representative of the germ $(\mathfrak{X}, 0)$ we can assume that the divisor $\bar{D}$ has a finite number of irreducible components, and every irreducible component of $\bar{D}$ intersects ${\widetilde{\nu_{\varphi}}}^{-1}(0)$. Thanks to the fact that each irreducible component $\bar{D}_{k}$ is mapped by the normalization map $n: \widetilde{\mathcal{N}_{\varphi} \mathfrak{X}} \rightarrow \mathcal{N}_{\varphi}(\mathfrak{X})$ to an irreducible component $D_{j}$ of $D=\left|\nu_{\varphi}^{-1}(W)\right|$ these conditions are also verified in $\mathcal{N}_{\varphi}(\mathfrak{X})$. 
Let $b \in \bar{D}$ be a point in the exceptional divisor lying over $W(0)$. Now, since $\bar{D}$ is a divisor, the ideal $J_{c}\left(J M_{\varphi}(F)_{\alpha}\right) \circ \widetilde{\nu_{\varphi}}$ is locally invertible, so at each $b \in \bar{D}(0)$ it is generated by a single element $g \circ \widetilde{\nu_{\varphi}}$, where $g \in J_{c}\left(J M_{\varphi}(F)_{\alpha}\right)$. By Proposition 7.7, we need to prove that for every such $b$ the function $M \circ \widetilde{\nu_{\varphi}}$ lies in the product $I\left(Y, \bar{D}_{k}\right) J_{c}\left(J M_{\varphi}(F)_{\alpha}\right) \circ \widetilde{\nu_{\varphi}}$, or equivalently (from the proof of the proposition) that the meromorphic function $k$ locally defined by $\frac{M \circ \widetilde{\nu_{\varphi}}}{g \circ \nu_{\varphi}}$ is holomorphic and vanishes at $b$ if $b$ lies over $(0,0) \in Y$.

Note that if $\widetilde{\nu_{\varphi}}(b)$ is not in $Y$ then the ideal $I\left(Y, \bar{D}_{k}\right) O_{\widetilde{\mathcal{N}_{\varphi} \mathfrak{x}, b}}$ is not a proper ideal and so all we need to prove is that $M \circ \widetilde{\nu_{\varphi}}$ belongs to the ideal $J_{c}\left(J M_{\varphi}(F)_{\alpha}\right) \circ \widetilde{\nu_{\varphi}}$, which by Proposition 7.7 is equivalent to $k$ being holomorphic and also to $M \in \overline{J_{c}\left(J M_{\varphi}(F)_{\alpha}\right)}$. Now, by Remark 8.3-1, for any point $(z, t) \in \mathfrak{X} \backslash \mathfrak{X}(0)$ we already have $M \in \overline{J_{c}\left(J M_{\varphi}(F)_{\alpha}\right)}$ which implies that the function $k$ is holomorphic on $\bar{D} \backslash \bar{D}(0)$, and so its polar locus is contained in $\bar{D}(0)$.

Let $(z, 0) \in W$ such that $(z, 0)$ is not in $\operatorname{Sing}_{\varphi} \mathfrak{X}$, that is $(z, 0)$ is a smooth point of both the space $\mathfrak{X}$ and the special fiber $\mathfrak{X}(0)$. Then, the vertical hyperplane $H=[0: \cdots: 0: 1] \in \breve{\mathbb{P}}^{n+1}$ cannot be tangent to $\mathfrak{X}$ at $(z, 0)$ and so by Remark 8.3-1 we have $M \in \overline{J_{c}\left(J M_{\varphi}(F)_{\alpha}\right)}$ and $k$ holomorphic. Indeed, if $H$ is tangent to $\mathfrak{X}$ at the point $(z, 0)$, then the point $(z, 0)$ is a singular point of $\mathfrak{X} \cap H=\mathfrak{X}(0)$. This implies that the polar locus of $k$ is contained in ${\widetilde{\nu_{\varphi}}}^{-1}(\operatorname{Sing} \mathfrak{X}(0))$, but by Corollary 8.6 the dimension of $\nu_{\varphi}^{-1}(\operatorname{Sing} \mathfrak{X}(0))$ is less than or equal to $d-1$, and since the normalization map is finite we also have $\operatorname{dim}{\widetilde{\nu_{\varphi}}}^{-1}(\operatorname{Sing} \mathfrak{X}(0))<d$, so it has codimension at least 2 . However, in a normal space the polar locus of a meromorphic function is of codimension 1 or empty ([8, Thm. 71.12, p. 307]), which implies that $k$ is holomorphic at every point $b \in \bar{D}$.

All that is left to prove is that the holomorphic function $k$ vanishes at every point $b \in \bar{D}$ lying over $Y$. Since for any point $y \neq 0 \in Y$ the pair $\left(\mathfrak{X}^{0}, Y\right)$ satisfies Whitney's condition a) at $y$ we have that $k$ vanishes on ${\widetilde{\nu_{\varphi}}}^{-1}(Y \backslash\{(0,0)\})$, and by continuity it vanishes on its closure in $\widetilde{\mathcal{N}_{\varphi} \mathfrak{X}}$. But by Lemma $8.7-2$ the aforementioned closure is equal to ${\widetilde{\nu_{\varphi}}}^{-1}(Y)$, and so we have that the function $k$ vanishes at any point $b$ lying over $(0,0) \in Y$.

Let $Z \subset \mathfrak{X}$ be the subspace defined by the ideal $I$ of 8.4 as before. Note that the key point in proving the previous proposition is the inequality dim $\nu_{\varphi}^{-1}(\operatorname{Sing} \mathfrak{X}(0))<d$ which was a consequence of 8.5 and this gives us the following result. 
Proposition 8.9. - Let $(X, 0) \subset\left(\mathbb{C}^{n+1}, 0\right)$ be a reduced and irreducible $d$ dimensional germ of an analytic singularity such that the tangent cone is reduced. Then $(X, 0)$ does not have exceptional cones if and only if $\nu_{\varphi}^{-1}(Z)$ does not have vertical components over $\mathfrak{X}(0)$.

Proof. - If $(X, 0)$ does not have exceptional cones, then it is Proposition 8.5. On the other hand, if $\nu_{\varphi}^{-1}(Z)$ does not have vertical components over $\mathfrak{X}(0)$ then Corollary 8.6 and the proof of Proposition 8.8 gives us that the pair $\left(\mathfrak{X}^{0}, Y\right)_{0}$ satisfies Whitney's condition a) at the origin, and by 6.5 this is equivalent to $(\mathfrak{X}, 0)$ having no exceptional cones. Finally, this implies that $(X, 0)$ does not have exceptional cones either.

REMARK 8.10. - Note that if $(X, 0)$ has exceptional cones then, $(\mathfrak{X}, 0)$ also has exceptional cones.

Indeed, if $\kappa_{\mathfrak{X}}: C(\mathfrak{X}) \rightarrow \mathfrak{X}$ is the conormal space of $\mathfrak{X}$ and $\kappa_{X}: C(X) \rightarrow X$ the conormal space of $X$, then $\kappa_{\mathfrak{X}}^{-1}(Y \backslash\{0\})=Y \backslash\{0\} \times \kappa_{X}^{-1}(0)$ and so $\kappa_{\mathfrak{X}}^{-1}(Y)$ contains $Y \times \kappa_{X}^{-1}(0)$. In particular, if $H=\left[a_{0}: \cdots a_{n}\right] \in \kappa_{X}^{-1}(0) \subset \check{\mathbb{P}}^{n}$, but $H$ is not tangent to the tangent cone $C_{X, 0}$, then $\widetilde{H}=\left[a_{0}: \cdots: a_{n}: 0\right] \in \kappa_{\mathfrak{X}}^{-1}(0) \subset$ $\check{\mathbb{P}}^{n+1}$ and it can not be tangent to the tangent cone $C_{\mathfrak{X}, 0}=C_{X, 0} \times \mathbb{C}$.

We can summarize all we have done so far with the following theorem:

TheOREM 8.11. - Let $(X, 0)$ be a reduced and equidimensional germ of a complex analytic singularity, and suppose that its tangent cone $C_{X, 0}$ is reduced. Then the following statements are equivalent:

1. The germ $(X, 0)$ does not have exceptional cones.

2. The pair $\left(\mathfrak{X}^{0}, Y\right)$ satisfies Whitney's condition a) at the origin.

3. The pair $\left(\mathfrak{X}^{0}, Y\right)$ satisfies Whitney's conditions a) and b) at the origin.

4. The germ $(\mathfrak{X}, 0)$ does not have exceptional cones.

Proof. - Let $(X, 0)=\bigcup_{i=1}^{r}\left(X_{i}, 0\right)$ be the irreducible decomposition of $(X, 0)$. Then by Corollary 8.1, and Lemma 2.5 it is enough to verify these equivalences for each irreducible component $\left(X_{j}, 0\right)$ and its specialization space $\left(\mathfrak{X}_{j}, 0\right)$. Now for an irreducible germ we have:

1) $\Rightarrow 2$ ) by Proposition 8.8 .

2) $\Rightarrow 3$ ) by Proposition 6.1 .

$3) \Rightarrow 4)$ by 6.5 .

$4) \Rightarrow 1$ ) by Remark 8.10 .

Suppose that $(X, 0)$ has as isolated singularity, but $C_{X, 0}$ does not, then:

1. Either the singular locus of $\mathfrak{X}(0)$ is contained in the singular locus of $\mathfrak{X}$ and so this last space has an irreducible component contained in the special fiber $\mathfrak{X}(0)$. 
2. Or, every point $p \in \operatorname{Sing} \mathfrak{X}(0)$ is smooth in $\mathfrak{X}$, which implies that the "vertical" hyperplane $H_{t}:=\{t=0\}$ is tangent to $\mathfrak{X}$ at $p$, and so $H_{t}$ is a limit of tangent hyperplanes to $(\mathfrak{X}, 0)$.

In any case, this will prevent us from building a Whitney stratification of $\mathfrak{X}$ having $Y$ as a stratum. This kind of phenomenom is quite general and has little to do with the isolated singularity case. So in order to be able to build the Whitney stratification we want, it is important to have some control on the behavior of the singular locus of $\mathfrak{X}$. The following lemma will help us manage this situation in the case of a complete intersection tangent cone.

Lemma 8.12. - Let $\nu_{\varphi}: \mathcal{N}_{\varphi}(\mathfrak{X}) \rightarrow \mathfrak{X}$ and $(Z, 0) \subset(\mathfrak{X}, 0)$ be defined by the ideal I of 8.4 as before. Let $D=\nu_{\varphi}^{-1}(Z)$ be the exceptional divisor. If $D$ does not have vertical components over $\mathfrak{X}(0)$, then set-theoretically, the closure of $Z \backslash Z(0)$ in $\mathfrak{X}$ is equal to $Z$.

Proof. - Let us consider the map $h:(Z, 0) \rightarrow(\mathbb{C}, 0)$. If $h$ is flat, we have nothing to prove, so suppose $\mathrm{h}$ is not flat. Then, we can find a minimal primary decomposition of $I$ in $O_{\mathfrak{X}, 0}$ :

$$
I=Q_{1} \cap Q_{2} \cap \cdots \cap Q_{s}
$$

such that $t^{n_{i}} \in Q_{i}$ for $1<r \leq i \leq s$ with $n_{i}>0$, so it corresponds to a possibly embedded irreducible component of the germ $(Z, 0)$ contained in the special fiber $Z(0)$.

Let $I=Q \cap B$, where $B=Q_{r} \cap \cdots \cap Q_{s}$. There exists a small neighbourhood of the origin $U \subset \mathfrak{X}$, such that $I(U)=Q(U) \cap B(U)$, and for every $x \in U$ we have the equality $I_{x}=Q_{x} \cap B_{x}$ in $O_{\mathfrak{X}, x}$. But, for any open set $V \subset U$ such that $0 \notin V$, since $t^{m} \in B(V)$ and $t^{m}$ is a unit in $O_{\mathfrak{X}}(V)$ we have that $I_{x}=Q_{x}$ in $O_{\mathfrak{X}, x}$ for any point $x \in Z \backslash\{0\}$, so their integral closures are equal $\overline{I_{x}}=\overline{Q_{x}}$ for every point $x \in V$.

Let $\widetilde{\nu_{\varphi}}: \overline{\mathcal{N}_{\varphi}(\mathfrak{X})} \stackrel{n}{\longrightarrow} \mathcal{N}_{\varphi}(\mathfrak{X}) \stackrel{\nu_{\varphi}}{\longrightarrow} \mathfrak{X}$ be the composition of $\nu_{\varphi}$ and the normalization of $\mathcal{N}_{\varphi}(\mathfrak{X})$. By hypothesis, $D$ does not have vertical components over the origin, and since the normalization is a finite map, we have that $\bar{D}={\widetilde{\nu_{\varphi}}}^{-1}(Z)=n^{-1}(D)$ does not have vertical components over the origin either. Let $w \in Q$, then for $U$ sufficiently small $w \in Q(U)$. Now, we know that the coherent ideal $\widetilde{I}:=I O_{\widetilde{\mathcal{N}_{\varphi}(\mathfrak{X})}}$ is locally invertible, so in particular for any point $p \in \bar{D}$ there exists an open neighborhood $V_{p}$ of $p$ in $\overline{\mathcal{N}_{\varphi}(\mathfrak{X})}$ such that $\widetilde{I}\left(V_{p}\right)=\left\langle g_{p}\right\rangle O_{\widetilde{\mathcal{N}_{\varphi}(\mathfrak{X})}}\left(V_{p}\right)$.

For any such neighborhood, we can consider the meromorphic function $q:=$ $\left(w \circ \widetilde{\nu_{\varphi}}\right) / g_{p}$. The polar locus of $q$ is contained in $\bar{D}$, more precisely, since the ideal $\widetilde{I}$ and $\widetilde{Q}$ coincide outside ${\widetilde{\nu_{\varphi}}}^{-1}(0)$, we have that the polar locus of $q$ is 
contained in ${\widetilde{\nu_{\varphi}}}^{-1}(Z(0))$. But $\bar{D}$ does not have vertical components over $\mathfrak{X}(0)$ so ${\widetilde{\nu_{\varphi}}}^{-1}(Z(0))$ is of codimension at least 2 . Since in a normal space the polar locus of a meromorphic function is of codimension one or empty ([8, Thm. 71.12,p. 307]), $q$ is actually holomorphic and $\widetilde{I}=\widetilde{Q}$ in $\overline{\mathcal{N}_{\varphi}(\mathfrak{X})}$, which implies by $[9$, Thm 2.1 , p. 799$]$ that the integral closures $\bar{I}=\bar{Q}$ are equal in $O_{\mathfrak{X}, 0}$.

Finally, since the integral closure of an ideal is contained in its radical, then set theoretically $Z$ is the zero locus of $\bar{I}$, that its $|Z|=V(\bar{I})=V(\bar{Q})=V(Q)$ and it does not have vertical components over the origin.

Suppose now that both $(X, 0)$ and its tangent cone are reduced complete intersections, then the specialization space $(\mathfrak{X}, 0)$ is also a complete intersection. In particular, refering back to Remark 7.10, there is no need to choose an $\alpha$, and the ideal $I$ of 8.4 can be chosen as the relative Jacobian ideal $J_{c}\left(J M_{\varphi}(F)\right)$ which set-theoretically defines the relative singular locus $\operatorname{Sing}_{\varphi} \mathfrak{X}$. Note that that the restriction of the ideal $J_{c}\left(J M_{\varphi}(F)\right)$ to the special fiber is equal to the Jacobian ideal $J_{C_{X, 0}}$ of the tangent cone $C_{X, 0}$ in $O_{C_{X, 0}}$. This implies that the strict transform of $\mathfrak{X}(0)$ with respect to this blowup is equal to the Nash modification $\mathcal{N} C_{X, 0}$ of the fiber.

Proposition 8.13. - Let $(X, 0) \subset\left(\mathbb{C}^{n+1}, 0\right)$ be a reduced germ of a singularity such that the tangent cone $C_{X, 0}$ is a reduced complete intersection. Let $\left|\operatorname{Sing} C_{X, 0}\right|=\bigcup E_{\alpha}$ be the irreducible decomposition of the singular locus of the tangent cone. If there exists an $\alpha$, such that $E_{\alpha}$ is not completely contained in the reduced tangent cone $\left|C_{|\mathrm{Sing} \mathrm{X}|, 0}\right|$, then it is contained in an exceptional cone. In particular we have the inclusion

$$
\mid \text { Sing } C_{X, 0}|\subset| C_{|\operatorname{Sing} \mathrm{X}|, 0} \mid \bigcup\{\text { Exceptional cones }\}
$$

Proof. - Let $\varphi:(\mathfrak{X}, 0) \rightarrow(\mathbb{C}, 0)$ be the specialization space of $X$ to its tangent cone $\left(C_{X, 0}, 0\right)$, and let $\nu_{\varphi}: \mathcal{N}_{\varphi}(\mathfrak{X}) \rightarrow \mathfrak{X}$ be considered as the blowup of $\mathfrak{X}$ with center $Z \subset \mathfrak{X}$ defined by the ideal $J_{c}\left(J M_{\varphi}(F)\right)$, and exceptional divisor $D \subset \mathcal{N}_{\varphi}(\mathfrak{X})$. Since set-theoretically $Z$ is the relative singular locus of $\mathfrak{X}$, then if we set $W$ as the closure of $Z \backslash Z(0)$ in $\mathfrak{X}$, then set theoretically $W(0)$ is $\left|C_{|\operatorname{Sing} \mathrm{X}|, 0}\right|$, so the existence of the $E_{\alpha}$ in the hypothesis amounts to $Z$ having a vertical (irreducible) component $Z_{\beta}$ over the origin.

The existence of such a $Z_{\beta}$ implies by 8.12 the existence of a vertical component $D_{\beta}$ of $|D|$, which then implies by 8.5 that the germ $(X, 0)$ has exceptional cones. Now for any point $z \in Z_{\beta} \backslash W$ there exists an open neighborhood $z \in U_{z} \subset \mathfrak{X}$ such that $U_{z} \cap W=\varnothing$ and $Z_{\beta} \backslash W$ is dense in $Z_{\beta}$. That is, there exists an open neighborhood $U$ of $Z_{\beta} \backslash W$ in $\mathfrak{X}$, such that $U \cap W=\varnothing$, and so $\nu_{\varphi}^{-1}(U \cap W)=\nu_{\varphi}^{-1}(U) \cap \nu_{\varphi}^{-1}(W)=\varnothing$. But $\nu_{\varphi}^{-1}(W)$ contains $\overline{D \backslash D(0)}$, and 
$\nu_{\varphi}^{-1}(U) \cap D$ is not empty, so there is necessarily an irreducible component $D_{\beta}$ of $D$, such that $D_{\beta} \supset \nu_{\varphi}^{-1}\left(Z_{\beta}\right)$ and $D_{\beta}$ is completely contained in $D(0)$. All that is left to prove is that the component $D_{\beta}$ is mapped by $\nu_{\varphi}$ into an exceptional cone.

By Remark 8.3 , the strict transform $\overline{\nu_{\varphi}^{-1}(\mathfrak{X}(0) \backslash Z)}$ is equal to the Nash modification of the fiber $\mathfrak{X}(0)$ which has dimension $d$, on the other hand since $D_{\beta}$ is an irreducible component of the divisor $D$ it is also of dimension $d$ and so cannot be contained in $\mathcal{N} \mathfrak{X}(0)$, i.e. $D_{\beta} \nsubseteq \mathscr{N} \mathfrak{X}(0)$.

Now, by [16, Proposition 2.1.4.1, p. 562], the cones of the aureole are set theoretically the images by $\kappa_{\varphi}$ of the irreducible components of $\left|\kappa_{\varphi}^{-1}(\mathfrak{X}(0))\right|$. So let us consider the relative version of the diagram given in Proposition 4.1, relating the relative Nash modification $\mathcal{N}_{\varphi} \mathfrak{X}$ with the relative conormal space $C_{\varphi}(\mathfrak{X})$.

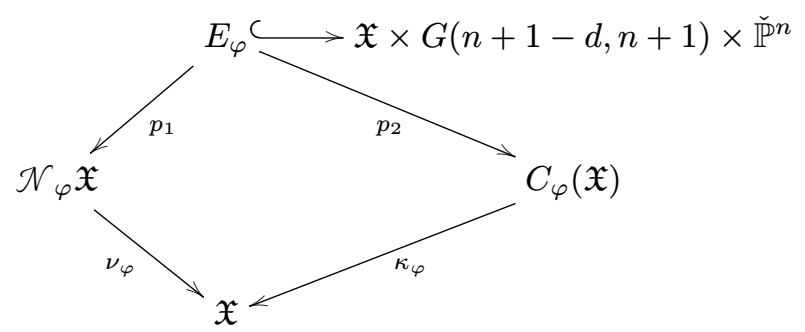

By commutativity of the diagram, we have the equality $p_{2}\left(p^{-1}(\mathcal{N} \mathfrak{X}(0))\right)=$ $C(\mathfrak{X}(0))$, where $C(\mathfrak{X}(0))$ denotes the conormal space of the fiber $\mathfrak{X}(0)$ and it is

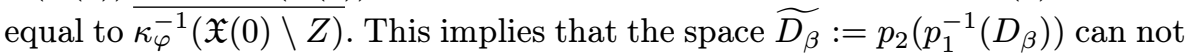
be contained in $C(\mathfrak{X}(0))$. Now, the conormal space $C(\mathfrak{X}(0))$ is of dimension $n$, and since $C_{\varphi}(\mathfrak{X}) \rightarrow \mathfrak{X} \rightarrow \mathbb{C}$ is isomorphic to the specialization space of $C(X)$ to its normal cone along $\kappa_{X}^{-1}(0)$ ([12, Lemma A.4.1, p. 190]), then the dimension of $\kappa_{\varphi}^{-1}(\mathfrak{X}(0))$ is also $n$. This means that $\widetilde{D_{\beta}}$ is contained in an irreducible component of $\left|\kappa_{\varphi}^{-1}(\mathfrak{X}(0))\right|$ outside of $C(\mathfrak{X}(0))$ and so is mapped by $\kappa_{\varphi}$ into an exceptional cone.

Note that we always have the inclusion $\left|C_{|\operatorname{Sing} \mathrm{X}|, 0}\right| \subset\left|\operatorname{Sing} C_{X, 0}\right|$, so the absence of exceptional cones together with 8.13 tells us that in this setting not only do the relative singular locus, and the singular locus of $\mathfrak{X}$ coincide, but also that $\overline{\operatorname{Sing} \mathfrak{X} \backslash \mathfrak{X}(0)}=\operatorname{Sing} \mathfrak{X}$. In particular we have $\left|C_{|\operatorname{Sing} X|, 0}\right|=\left|\operatorname{Sing} C_{X, 0}\right|$ and this leaves us in a good position to continue building a Whitney stratification of $\mathfrak{X}$ having $Y$ as a stratum. 
Corollary 8.14. - Let $(X, 0)$ satisfy the hypothesis of Theorem 8.11. If $(X, 0)$ has an isolated singularity and its tangent cone is a complete intersection singularity, then the absence of exceptional cones implies that $C_{X, 0}$ has an isolated singularity and $\{\mathfrak{X} \backslash Y, Y\}$ is a Whitney stratification of $\mathfrak{X}$.

Proof. - Proposition 8.13 tells us that $\left|C_{\mid \operatorname{Sing~X|,0}}\right|=\left|\operatorname{Sing} C_{X, 0}\right|$, and since $(X, 0)$ has an isolated singularity then $\left|C_{|\operatorname{Sing} X|, 0}\right|=\{0\}$ and so the tangent cone $\left(C_{X, 0}, 0\right)$ also has an isolated singularity. This implies, that Sing $\mathfrak{X}=Y$, and Theorem 8.11 finishes the proof.

There is a partial converse to the corollary, in which we can construct a Whitney stratification of $\mathfrak{X}$ under the assumption that the tangent cone has an isolated singularity at the origin.

COROLlary 8.15. - Let $(X, 0)$ satisfy the hypothesis of Theorem 8.11. If the tangent cone $\left(C_{X, 0}, 0\right)$ has an isolated singularity at the origin, then $(X, 0)$ has an isolated singularity and $\{\mathfrak{X} \backslash Y, Y\}$ is a Whitney stratification of $\mathfrak{X}$.

Proof. - The first step is to prove that $(X, 0)$ does not have exceptional cones, however by [16, Prop. 2.1.4.2, p. 563] this is always the case when the tangent cone has an isolated singularity at the origin.

Now, by Theorem 8.11, it is enough to prove that the singular locus of $\mathfrak{X}$ is $Y$. It is a general fact that the relative singular locus $\operatorname{Sing}_{\varphi} \mathfrak{X}$ of $\mathfrak{X}$, contains the singular locus Sing $\mathfrak{X}$, and they coincide away from the special fiber. In other words, the space $W:=\operatorname{Sing}_{\varphi} \mathfrak{X} \backslash\{\mathfrak{X}(0)\}$ is isomorphic via $\phi: \mathfrak{X} \backslash \mathfrak{X}(0) \rightarrow X \times \mathbb{C}^{*}$ to $\operatorname{Sing} X \times \mathbb{C}^{*}$, and so the map induced by $\varphi$ to its closure $\bar{W} \rightarrow \mathbb{C}$ can be identified with the specialization space of $|\operatorname{Sing} X|$ to its tangent cone. In view of this, the hypothesis tells us that the only singular point of $\mathfrak{X}$ in the special fiber is the origin $(0,0)$; this implies $\bar{W}(0)=\{0\}$ and since it is isomorphic to the tangent cone $C_{|\operatorname{Sing} X|, 0}$, then $(X, 0)$ has an isolated singularity and Sing $\mathfrak{X}=Y$ which finishes the proof.

EXAMPle 8.1. - Let $(V, 0) \subset\left(\mathbb{C}^{n+1}, 0\right)$ be a reduced and irreducible isolated complete intersection variety defined by an homogeneous ideal $I_{0}=$ $\left\langle h_{m_{1}}, \ldots, h_{m_{k}}\right\rangle$, where $m_{i}$ is the degree of the polynomial. That is, $V$ is the cone over a smooth, complete intersection, projective variety.

Let $(X, 0) \subset\left(\mathbb{C}^{n+1}, 0\right)$ be the germ defined by the ideal $I=\left\langle h_{1}, \ldots, h_{k}\right\rangle$, where $h_{i}=h_{m_{i}}+P_{i}$ and $P_{i} \in \mathbb{C}\left\{z_{0}, \ldots, z_{n}\right\}$ is such that $\operatorname{ord}_{0} P_{i}(z)>m_{i}$. Then:

- The germ $(X, 0)$ is a reduced complete intersection.

- The tangent cone $C_{X, 0}$ is defined by the ideal $I_{0}$ and so it is isomorphic to $V$. 
That $X$ is a complete intersection can be seen by considering the analytic family $\left\{X_{t}\right\}$ defined by the $h_{i}^{t}:=h_{m_{i}}+t P_{i}$ and the upper semicontinuity of fiber dimension. For the other assertion consider the radical idea $\widetilde{I}:=\sqrt{I}$ defining $|X|$. This gives us the following inclusion of initial ideals

$$
\operatorname{In}_{\mathfrak{M}} I_{0}=I_{0} \subset \operatorname{In}_{\mathfrak{M}} I \subset \operatorname{In}_{\mathfrak{M}} \widetilde{I}
$$

and as a result the surjective morphism of analytic algebras:

$$
\begin{aligned}
\frac{\mathbb{C}\left\{z_{0}, \ldots, z_{n}\right\}}{I_{0}} & \longrightarrow \frac{\mathbb{C}\left\{z_{0}, \ldots, z_{n}\right\}}{\operatorname{In}_{\mathfrak{M}} \widetilde{I}} \\
O_{V, 0} & \longrightarrow O_{C_{|X|, 0}}
\end{aligned}
$$

But $V$ is irreducible, so $O_{V, 0}$ is an integral domain and since both algebras have krull dimension $n+1-k$ they are isomorphic and $I_{0}=\operatorname{In}_{\mathfrak{M}} \widetilde{I}$. Finally, this tells us that $\operatorname{In}_{\mathfrak{M}} \widetilde{I}=\left\langle\operatorname{In}_{\mathfrak{M}} h_{1}, \ldots, \operatorname{In}_{\mathfrak{M}} h_{k}\right\rangle$, which implies that $\widetilde{I}=\left\langle h_{1}, \ldots, h_{k}\right\rangle=I$ and so $X$ is reduced and $C_{X, 0}=V$.

Now, by construction, the specialization space $\varphi: \mathfrak{X} \rightarrow \mathbb{C}$ is defined by the equations $H_{i}(z, t)=t^{-m_{i}} h_{i}(t z)$ in $\mathbb{C}^{n+1} \times \mathbb{C}$ and since the tangent cone $C_{X, 0}$ is reduced and has an isolated singularity at the origin, Corollary 8.15 tells us that $\{\mathfrak{X} \backslash Y, Y\}$ is a Whitney stratification of $\mathfrak{X}$.

\section{Conclusion}

We have verified that the absence of exceptional cones allows us to start building a Whitney stratification of $\mathfrak{X}$ having $Y$ as a stratum. The question now is how to continue. Proposition 8.13 tells us, at least in the complete intersection case, that the singular locus of $\mathfrak{X}$ coincides with the specialization space $Z$ of $|\operatorname{Sing} X|$ to its tangent cone.

Suppose now, that the germ $(|\operatorname{Sing} X|, 0)$ has a reduced tangent cone, then a stratum $\mathfrak{X}_{\lambda}$ containing a dense open set of $Z$ will satisfy Whitney's conditions along $Y$ if and only if the germ ( $|\operatorname{Sing} X|, 0)$ does not have exceptional cones.

In view of this it seems reasonable to start by assuming the existence of a Whitney stratification $\left\{X_{\lambda}\right\}$ of $(X, 0)$ such that for every $\lambda$ the germ $\left(\overline{X_{\lambda}}, 0\right)$ has a reduced tangent cone and no exceptional cones. In this case, the specialization space $Z_{\lambda}$ of $\overline{X_{\lambda}}$ is canonically embedded as a subspace of $\mathfrak{X}$, and the partition of $\mathfrak{X}$ associated to the filtration given by the $Z_{\lambda}$ is a good place to start looking for the desired Whitney stratification of $\mathfrak{X}$ 


\section{BIBLIOGRAPHY}

[1] T. GAFFNEY - "Integral closure of modules and Whitney equisingularity", Invent. Math. 107 (1992), p. 301-322.

[2] _ "Aureoles and integral closure of modules", in Stratifications, singularities and differential equations, II (Marseille, 1990; Honolulu, HI, 1990), Travaux en Cours, vol. 55, Hermann, 1997, p. 55-62.

[3] T. Gaffney \& S. L. Kleiman - "Specialization of integral dependence for modules", Invent. Math. 137 (1999), p. 541-574.

[4] M. Gerstenhaber - "On the deformation of rings and algebras. II", Ann. of Math. 84 (1966), p. 1-19.

[5] G.-M. Greuel, C. Lossen \& E. Shustin - Introduction to singularities and deformations, Springer Monographs in Math., Springer, 2007.

[6] G.-M. Greuel \& G. Pfister - A singular introduction to commutative algebra, Springer, 2007.

[7] T. DE Jong \& G. Pfister - Local analytic geometry, Advanced Lectures in Mathematics, Friedr. Vieweg \& Sohn, 2000.

[8] L. KAUP \& B. KAUP - Holomorphic functions of several variables, de Gruyter Studies in Mathematics, vol. 3, Walter de Gruyter \& Co., 1983.

[9] M. Lejeune-Jalabert \& B. Teissier - "Clôture intégrale des idéaux et équisingularité", Ann. Fac. Sci. Toulouse Math. 17 (2008), p. 781-859.

[10] V. Navarro Aznar - "Conditions de Whitney et sections planes", Invent. Math. 61 (1980), p. 199-225.

[11] A. NobILE - "Some properties of the Nash blowing-up", Pacific J. Math. 60 (1975), p. 297-305.

[12] C. SABBAH - "Quelques remarques sur la géométrie des espaces conormaux", Astérisque 130 (1985), p. 161-192.

[13] B. TEISSIER - "Cycles évanescents, sections planes et conditions de Whitney", Astérisque 7-8 (1973), p. 285-362.

[14] L. D. Ung TrÁng - "Limites d'espaces tangents sur les surfaces", Nova Acta Leopoldina (N.F.) 52 (1981), p. 119-137.

[15] L. D. ung Tráng \& B. Teissier - "Sur la géométrie des surfaces complexes. I. Tangentes exceptionnelles", Amer. J. Math. 101 (1979), p. 420452.

[16] L. D. TrÁng \& B. Teissier - "Limites d'espaces tangents en géométrie analytique", Comment. Math. Helv. 63 (1988), p. 540-578.

[17] H. Whitney - "Tangents to an analytic variety", Ann. of Math. 81 (1965), p. $496-549$. 